\title{
PAPER
}

\section{A large probability averaging theorem for the defocusing NLS}

To cite this article: D Bambusi et al 2019 Nonlinearity 323661

View the article online for updates and enhancements. 


\title{
A large probability averaging theorem for the defocusing NLS
}

\author{
D Bambusi ${ }^{1} \odot$, A Maiocchi $\odot$ and L Turri
}

Dipartimento di Matematica, Università degli Studi di Milano, Via Saldini 50, I-20133, Milano, Italy

E-mail: dario.bambusi@unimi.it, alberto.maiocchi@unimi.it and luca.turri@unimi.it

Received 4 June 2018

Accepted for publication 10 April 2019

Published 30 August 2019

Recommended by Professor Dmitry V Treschev

\begin{abstract}
We consider the nonlinear Schrödinger equation on the one dimensional torus, with a defocusing polynomial nonlinearity and study the dynamics corresponding to initial data in a set of large measures with respect to the Gibbs measure. We prove that along the corresponding solutions the modulus of the Fourier coefficients is approximately constant for times of order $\beta^{2+\varsigma}$, $\beta$ being the inverse of the temperature and $\varsigma$ a positive number (we prove $\varsigma=1 / 10$ ). The proof is obtained by adapting to the context of Gibbs measure for PDEs some tools of Hamiltonian perturbation theory.
\end{abstract}

Keywords: Hamiltonian PDEs, Gibbs measure, averaging theory Mathematics Subject Classification numbers: 37K55

\section{Introduction and statement of the main result}

In this paper we study the dynamics of the defocusing NLS with a polynomial nonlinearity. We show that, with large probability in the sense of Gibbs measure, each of the actions of the unperturbed system is approximately invariant for long times. This is obtained by generalizing to the context of PDEs some tools of perturbation theory in Gibbs measure developed in recent years in the context of lattice dynamics $[3,10,11,13,18]$.

The system we consider is the defocusing NLS on the torus

$$
\mathrm{i} \dot{\psi}=-\Delta \psi+F^{\prime}\left(|\psi|^{2}\right) \psi, x \in \mathbb{T},
$$

\footnotetext{
${ }^{1}$ Author to whom any correspondence should be addressed.
} 
where $F$ is a polynomial of degree $q \geqslant 2, F(x):=\sum_{j=2}^{q} c_{j} x^{j}$, s.t. $F(x) \geqslant 0$ for any $x \geqslant 0$ and $c_{2}>0$. The flow of (1) is almost surely globally well-posed on any one of the spaces $H^{s}$ with $s$ fulfilling $\frac{1}{2}-\frac{1}{q-1}<s<\frac{1}{2}$ (see e.g. [6, 8], see also [12]). We fix $s$ in this range once for all.

We recall that the Gibbs measure is formally defined by

$$
\mathrm{d} \mu_{\beta}=\frac{\mathrm{e}^{-\beta\left(H(\psi)+\frac{1}{2}\|\psi\|_{L^{2}}^{2}\right)}}{Z(\beta)}, \beta>0, \quad Z(\beta):=\int_{H^{s}} \mathrm{e}^{-\beta\left(H(\psi)+\frac{1}{2}\|\psi\|_{L^{2}}^{2}\right)} \mathrm{d} \psi \mathrm{d} \bar{\psi},
$$

where $H$ is the Hamiltonian of the NLS (see (6)) and $\beta$ plays the role of the inverse of the temperature (we add the $L^{2}$-norm in order to avoid problems related to zero frequency). We study the system in the limit of $\beta$ large.

We denote by $\psi_{\mathrm{k}}$ the $\mathrm{k}$ th Fourier coefficients of $\psi$ defined by $\psi_{\mathrm{k}}:=\frac{1}{\sqrt{2 \pi}} \int_{0}^{2 \pi} \psi(x) \mathrm{e}^{-\mathrm{ikx} x} \mathrm{~d} x$.

Our main result is the following one.

Theorem 1.1. There exists $\beta^{*}>1, C, C^{\prime}>0$ s.t. for any $\eta_{1}, \eta_{2}>0$, any $\beta$ fulfilling

$$
\beta>\max \left\{\beta_{*}, \frac{C}{\eta_{1}^{\frac{10}{7}} \eta_{2}^{\frac{5}{7}}}\right\}
$$

and any $\mathrm{k} \in \mathbb{Z}$, there exists a measurable set $\mathcal{\partial}_{\mathrm{k}} \subset H^{s}$ whose complement $\mathrm{\partial}_{\mathrm{k}}^{c}$ has a small measure, namely $\mu_{\beta}\left(\mathcal{J}_{\mathrm{k}}^{c}\right)<\eta_{2}$ s.t., if the initial datum $\psi(0) \in \mathcal{J}_{\mathrm{k}}$ then the solution exists globally in $H^{s}$ and one has

$$
\left|\frac{\left|\psi_{\mathrm{k}}(t)\right|^{2}-\left|\psi_{\mathrm{k}}(0)\right|^{2}}{\left[\left(1+\mathrm{k}^{2}\right) \beta\right]^{-1}}\right|<\eta_{1}, \forall|t|<C^{\prime} \eta_{1} \sqrt{\eta_{2}} \beta^{2+\varsigma}, \varsigma=\frac{1}{10} .
$$

The following corollary gives a control of all the actions at the same time.

Corollary 1.2. Under the same assumption of theorem 1.1 and for any $\alpha<1 / 2$, there exists a measurable set $\mathcal{J}_{\alpha} \subset H^{s}$ with $\mu_{\beta}\left(\mathcal{J}_{\alpha}^{c}\right)<\eta_{2}$ s.t., if the initial datum $\psi(0) \in \mathcal{J}_{\alpha}$ then the solution exists globally in $H^{s}$ and one has

$\left|\frac{\left|\psi_{\mathrm{k}}(t)\right|^{2}-\left|\psi_{\mathrm{k}}(0)\right|^{2}}{\left[\left(1+\mathrm{k}^{2}\right)^{\alpha} \beta\right]^{-1}}\right|<\eta_{1}, \forall|t|<C^{\prime} \eta_{1} \sqrt{\eta_{2}} \beta^{2+\varsigma}, \quad \forall \mathrm{k} \in \mathbb{Z}, \quad \varsigma=\frac{1}{10}$.

Remark 1. The expectation value of $\psi_{\mathrm{k}}$ is $C_{1} / \sqrt{\left(1+\mathrm{k}^{2}\right) \beta}$, with a suitable constant $C_{1}$. Theorem 1.1 shows that with large probability with respect to the Gibbs measure, for large $\beta$ and for large times, the single $\mathrm{k}$-action changes very little along the motion. Take for example,

$$
\eta_{1}=\eta_{2}^{1 / 2} \text { and } \beta=C \eta_{2}^{-20} \gg \beta^{*},
$$

we get that $\mu_{\beta}\left(\mathcal{J}_{\mathrm{k}}^{c}\right)<\frac{C}{\beta^{1 / 20}}$ and for all initial data $\psi(0) \in \mathcal{J}_{\mathrm{k}}$ one has

$$
\left|\frac{\left|\psi_{\mathrm{k}}(t)\right|^{2}-\left|\psi_{\mathrm{k}}(0)\right|^{2}}{\left[\left(1+\mathrm{k}^{2}\right) \beta\right]^{-1}}\right|<\frac{C}{\beta^{1 / 40}}, \forall|t|<C^{\prime} \beta^{2+\frac{1}{20}} .
$$

Remark 2. The quantity $\left|\psi_{\mathrm{k}}\right|^{2}$ appears since it is the action of the linearized system. Theorem 1.1 shows that, for general initial data, $\left|\psi_{\mathrm{k}}\right|^{2}$ moves very little compared to its typical size 
over a timescale of order $\beta^{2+\varsigma}$. Corollary 1.2 controls all the actions at the same time at the prize of giving a slightly worse control on the actions with a large index.

Remark 3. If one considers (1) as a perturbation of the cubic integrable NLS, then one has that the main term of the perturbation is (in the equation) $|\psi|^{4} \psi$ whose size can be thought to be of order $\beta^{-5 / 2}$ which is of order $\beta^{-2}$ smaller then the linear part. For this reason one can think that the effective perturbation is of size $\beta^{-2}$. So one expects to obtain a control of the dynamics of the actions over a timescale of order $\beta^{2}$.

Theorem 1.1, not only gives a rigorous proof of this fact, but also shows that the actions remain approximately constant over a longer timescale. We do not expect the value of $\varsigma$ to be optimal.

Remark 4. In order to cover times longer than $\beta^{-2}$, we have to face the problem of small denominators. Indeed, over the longer timescale, the nonlinear corrections to the frequencies become relevant and the heart of the proof consists in giving an estimate of the measure of the phase space in which the nonlinear frequencies are nonresonant.

Theorem 1.1 is essentially an averaging theorem for perturbations of a linear resonant system.

We recall that previous results giving long-time stability of the actions in (1) have been obtained in [1] and [7]. The first two results allow us to control the dynamics for exponentially long times, but only for initial data close in the energy norm to some finite dimensional manifold, so essentially for a very particular set of initial data. Bourgain [7] was able to exploit the nonlinear modulation of the frequencies in order to show that for most (in a suitable sense, not related to Gibbs measure) initial data in $H^{s}$ with $s \gg 1$ the Sobolev norm of the solution is controlled for times longer then any inverse power of the small parameter.

Nothing is known for solutions with low regularity as those dealt with in the present paper.

Our result can be compared also to the result of Huang Guan [15], who proved a large probability averaging theorem for perturbations of $\mathrm{KdV}$ equation. We emphasize that the result of [15] deals with the quite artificial case in which the perturbation is smoothing, namely it maps functions with some regularity into functions with higher regularity. In our case we deal with the natural local perturbation given by a polynomial in $\psi$. Furthermore [15] only deals with smooth solution. We also recall [16] in which a weaker version of averaging theorem is obtained for solutions of some NLS-type equations. In that paper the initial datum is required to be more regular that in theorem 1.1 and the times covered are shorter.

Finally we mention the papers $[2,4,5]$ which deal with very smooth initial data and perturbations of nonresonant linear system. These results are clearly in a context very different from ours.

The proof of our result is based on the generalization to the context of Gibbs measure for PDEs of Poincaré's method of construction of approximate integrals of motion [14, 19]. The standard way of using this method consists in first using a formal algorithm giving the construction of objects which are expected to be approximate integrals of motion and then adding estimates in order to show that this actually happens. This is the way we proceed.

So, first, we develop a formal scheme of construction of the approximate integrals of motion which is slightly different from the standard one. This is due to the fact that the linearized system is completely resonant and we have to find a way to use the nonlinear modulation of the frequencies in order to control each one of the actions. We have also to restrict our construction to the region of the phase space in which the frequencies are nonresonant. This is obtained by eliminating (through cutoff functions) the regions of the phase space where the 
linear combinations of the frequencies that are met along the construction are smaller than $\delta$, where $\delta$ is a parameter that will be determined at the end of the construction.

The formal construction is contained in section 4. As a result of this section, for any $\mathrm{k}$, we obtain a function $\Phi_{\mathrm{k}}(\psi)$ close to $\left|\psi_{\mathrm{k}}\right|^{2}$ which is expected to be an approximate integral of motion.

The second step of the proof consists in estimating the $L^{2}\left(\mu_{\beta}\right)$ norm of $\dot{\Phi}_{\mathrm{k}}$ and in showing that it is small. To this end, we first prove that all the estimates can be done by working with the Gaussian measure associated to the linearized system, then we introduce the class of functions which will be needed for the construction. Then we show how to control the $L^{2}\left(\mu_{\beta}\right)$ norm of such functions. This is obtained by exploiting the decay of the Fourier modes of functions in the support of the Gibbs measure. Then we use similar ideas in order to show that the integral of a function of our class on the resonant region is small with $\delta$. Then we choose $\delta$ in order to minimize the $L^{2}\left(\mu_{\beta}\right)$ norm of $\dot{\Phi}_{\mathrm{k}}$, and we use the invariance of the Gibbs measure and Chebyshev theorem in order to pass from the estimate of $\dot{\Phi}_{\mathrm{k}}$ to the estimate of $\left|\Phi_{\mathrm{k}}(t)-\Phi_{\mathrm{k}}(0)\right|$. Finally, we show that this implies the control of $\left|\psi_{\mathrm{k}}\right|^{2}$.

\section{Preliminaries}

Explicitly, the Hamiltonian of (1) is given by

$$
H=H_{2}+P
$$

where

$$
\begin{aligned}
& H_{2}:=\frac{1}{2} \int_{0}^{2 \pi}|\nabla \psi(x)|^{2} \mathrm{~d} x, \\
& P=\sum_{j=2}^{q} H_{2 j}, H_{2 j}:=\frac{c_{j}}{2 j} \int_{0}^{2 \pi}|\psi(x)|^{2 j} \mathrm{~d} x .
\end{aligned}
$$

We will denote by $\Phi_{\mathrm{NLS}}^{t}$ its flow (see [9]). We consider the Gibbs measure $\mu_{\beta}$ associated to this Hamiltonian, which is known to be invariant with respect to $\Phi_{\mathrm{NLS}}^{t}[6,17,20,21]$ and that is formally defined in (2).

Given a function $f: H^{s} \rightarrow \mathbb{C}, f \in L^{2}\left(H^{s}, \mu_{\beta}\right)$, we define its average and its $L^{2}$-norm with respect to the measure $\mu_{\beta}$ as:

$$
\begin{aligned}
& \langle f\rangle:=\int_{H^{s}} f \mathrm{~d} \mu_{\beta} \\
& \|f\|_{\mu_{\beta}}^{2}:=\int_{H^{s}}|f|^{2} \mathrm{~d} \mu_{\beta} .
\end{aligned}
$$

Remark 5. From the invariance of $\mu_{\beta}$, one has that the average $\langle f\rangle$ and the $L^{2}$-norm $\|f\|_{\mu_{\beta}}$ of the functions are preserved along the flow, namely $\left\langle f \circ \Phi_{\mathrm{NLS}}^{t}\right\rangle=\langle f\rangle,\left\|f \circ \Phi_{\mathrm{NLS}}^{t}\right\|_{\mu_{\beta}}=\|f\|_{\mu_{\beta}}$ for any $t$.

From now on, we shall work using the Fourier coordinates. In these coordinates, $\mathrm{H}_{2}$ becomes 


$$
H_{2}:=\frac{1}{2} \sum_{k} k^{2}\left|\psi_{k}\right|^{2} .
$$

We give now some results on the relationship of the Gaussian measure with the Gibbs measure. Define the $H^{1}$-norm:

$$
\|\psi\|_{H^{1}}^{2}:=\sum_{k}\left(1+k^{2}\right)\left|\psi_{k}\right|^{2},
$$

then we can express $H_{2}+\frac{1}{2}\|\psi\|_{L^{2}}^{2}=\frac{1}{2}\|\psi\|_{H^{1}}^{2}$ and the Gaussian measure is formally defined by

$$
\mathrm{d} \mu_{g, \beta}:=\frac{\mathrm{e}^{-\frac{\beta}{2}\|\psi\|_{H^{1}}^{2}}}{Z_{g}(\beta)},
$$

with

$$
Z_{g}(\beta):=\int_{H^{s}} \mathrm{e}^{-\frac{\beta}{2}\|\psi\|_{H^{1}}^{2}} \mathrm{~d} \psi \mathrm{d} \bar{\psi} .
$$

Given a function $f: H^{s} \rightarrow \mathbb{C}$, we denote by

$$
\|f\|_{g, \beta}^{2}:=\int_{H^{s}}|f|^{2} \mathrm{~d} \mu_{g, \beta}
$$

its $L^{2}$-norm respect to $\mu_{g, \beta}$.

The following lemmas will be proved in the appendix A.

Lemma 2.1. There exists $\beta^{*}, \tilde{C}>0$ s.t. for any $\beta>\beta^{*}$ and for any function $f \in L^{2}\left(H^{s}, \mu_{g, \beta}\right)$, one has:

$$
\|f\|_{\mu_{\beta}} \leqslant\|f\|_{g, \beta} \mathrm{e}^{\tilde{C}} .
$$

We emphasize that the constant $\tilde{C}$ is independent of $\beta$ and $q$, where $q$ is the degree of the polynomial $F$ (see (1)).

Lemma 2.2. There exists $C_{\mathrm{sob}}, D^{\prime}>0$ s.t. for any $\beta>0$ and any function $f \in L^{2}\left(H^{s}, \mu_{g, \beta}\right)$, one has

$$
\|f\|_{\mu_{\beta}} \geqslant \mathrm{e}^{-\frac{C_{\text {sob }}}{2 \beta} q \max _{j} c_{j} D^{\prime j}}\left\|f \chi_{\left\{\|\psi\|_{H^{s_{1}}}<\frac{D^{\prime}}{\beta}\right\}}\right\|_{g, \beta}
$$

where $\chi_{\{U\}}(\psi)$ is the characteristic function of the set $U$.

The next lemma shows that every moment of $\mu_{\beta}$ is well defined.

Lemma 2.3. There exists $\beta^{*}>0$ s.t., for any $s_{1}<\frac{1}{2}, n \in \mathbb{N}, \beta>\beta^{*}$, one has

$$
\left\|\psi^{n}\right\|_{H^{s_{1}}} \in L^{1}\left(H^{s}, \mu_{\beta}\right) \cap L^{1}\left(H^{s}, \mu_{g, \beta}\right) .
$$

Finally, for the special case of the function $\left|\psi_{\mathrm{k}}\right|^{2}$, we have the following lemma.

Lemma 2.4. There exists $\beta^{*}>0, C>0$ s.t. for any $\beta>\beta^{*}$ s.t.

$$
\left\|\left|\psi_{\mathrm{k}}\right|^{2}\right\|_{\mu_{\beta}} \geqslant \frac{C}{\beta\left(1+\mathrm{k}^{2}\right)} .
$$




\section{Polynomials with frequency dependent coefficients}

In this section we introduce a class of function on $H^{s}$, which is stable under the perturbative construction and we prove some results needed for the rest of the proof.

Definition 1. Let $B_{1}, B_{2}$ be two Banach spaces, we say that $F(y): B_{1} \rightarrow B_{2}$ is a polynomial of degree $n$ if there exists a $n$-multilinear form $\tilde{F}$ s.t. for any $y \in B_{1}$, one has $F(y)=\tilde{F}(\underbrace{y, y, \ldots, y}_{n})$.

Remark 6. In particular a polynomial $f: H^{s} \rightarrow \mathbb{C}$ of degree $n$ has the form:

$$
f(\psi)=\sum_{l, m} \psi^{l} \bar{\psi}^{m} f_{l, m}
$$

where $l=\left\{l_{k}\right\}, m=\left\{m_{k}\right\}, l_{k}, m_{k} \in \mathbb{N}, \sum_{k} l_{k}+m_{k}=n, f_{l, m} \in \mathbb{C}, \psi^{l}=\ldots \psi_{-k}^{l_{-k}} \ldots \psi_{k}^{l_{k}} \ldots$ and the same for $\bar{\psi}^{m}$.

Definition 2. We say that a polynomial $f$ of the form (8) of degree $2 n$ is of class $P_{2 n}$ if it fulfills the null momentum condition, i.e.

$$
f_{l, m} \neq 0 \text { only if } \sum_{k \in \operatorname{Supp}(l)} k=\sum_{k \in \operatorname{Supp}(m)} k \text { and } \sum_{k} l_{k}=\sum_{k} m_{k}=n .
$$

On $P_{2 n}$, we introduce the following norm

$$
|| f||\left|:=\sup _{l, m}\right| f_{l, m} \mid .
$$

Remark 7. In the following, due to (9), we will write a polynomial $f \in P_{2 n}$ also in the equivalent following form, more convenient in a lot of situations

$$
f(\psi)=\sum_{\substack{k=\left(k_{1} \ldots k_{2 n}\right) \\ \sum_{i=1}^{n} k_{i}=\sum_{i=n+1}^{2 n} k_{i}}} f_{k} \prod_{i=1}^{n} \psi_{k_{i}} \bar{\psi}_{k_{i+n}} .
$$

The next lemma shows that the polynomials of class $P_{2 n}$ are smooth polynomials on $H^{s_{1}}$, $\frac{1}{2}-\frac{1}{n}<s_{1}<\frac{1}{2}$.

Lemma 3.1. Let $n$ be a positive integer and $s_{1}$ s.t. $\frac{1}{2}-\frac{1}{2 n}<s_{1}<\frac{1}{2}, f \in P_{2 n}$, then there exists $C\left(s_{1}, n\right)>0$ s.t.

$$
|f(\psi)| \leqslant C\left(s_{1}, n\right)\|\psi\|_{H^{s_{1}}}^{2 n}|| f|\||
$$

\section{Proof.}

$$
\begin{aligned}
|f(\psi)| \leqslant & \sum_{\substack{k_{1}, \ldots, k_{2 n} n \\
\sum_{i=1}^{n} k_{i}=\sum_{i=n+1}^{2 n} k_{i}}}\left|f_{k_{1}, \ldots, k_{2 n}}\right| \prod_{i=1}^{2 n}\left|\psi_{k_{i}}\right| \\
\leqslant & \left\|f\left|\| \sum_{\substack{k_{1}, \ldots, k_{2 n} n \\
\sum_{i=1}^{n} k_{i}=\sum_{i=n+1}^{2 n} k_{i}}} \prod_{i=1}^{2 n}\right| \psi_{k_{i}} \mid .\right.
\end{aligned}
$$


We define $\varphi:=\left\{\varphi_{k}\right\}:=\left\{\left|\psi_{k}\right|\right\}, \tilde{\varphi}:=\sum_{k} \varphi_{k} \mathrm{e}^{\mathrm{i} k x}$, so, using Sobolev's embedding $H^{s_{1}} \subset L^{2 n}$ for $\frac{1}{2}-\frac{1}{2 n}<s_{1}<\frac{1}{2}$, one has:

$$
\begin{aligned}
|f(\psi)| & \leqslant\left\|f\left|\left\|\sum_{\substack{k_{1}, \ldots k_{2 n} \\
\sum_{i=1}^{n} k_{i}=\sum_{i=n+1}^{2 n} k_{i}}} \prod_{i=1}^{2 n} \varphi_{k_{i}}=\right\| \tilde{\varphi}\left\|_{L^{2 n}}^{2 n}\right\| f\right|\right\| \\
& \leqslant C\left(s_{1}, n\right)\|\tilde{\varphi}\|_{H^{s_{1}}}^{2 n}\left|\left\|f \left|\left\|=C\left(s_{1}, n\right)\right\| \psi\left\|_{H^{s_{1}}}^{2 n} \mid\right\| f\|\| .\right.\right.\right.
\end{aligned}
$$

We will also consider the functions $f \in C^{r}\left(\ell^{1}, P_{2 n}\right), f: \ell^{1} \ni \omega=\left\{\omega_{j}\right\} \rightarrow f(\psi, \omega)=$ $\sum_{\substack{k=\left(k_{1} \ldots, k_{2 n}\right) \\ \sum_{i=1}^{n} k_{i}=\sum_{i=n+1}^{2 n} k_{i}}} f_{k}(\omega) \prod_{i=1}^{n} \psi_{k_{i}} \bar{\psi}_{k_{i+n}}$. In the following $\omega_{j}$ will be the nonlinear modulation of the $j$ th frequency.

Actually we need to keep the information of the size of the different derivatives of $f$. So, we give the following definition.

Definition 3. We will say that $f \in P^{r}\left(2 n,\left\{A_{i}\right\}_{i=0}^{r}\right)$ if $f \in C^{r}\left(\ell^{1}, P_{2 n}\right)$ and

$$
\sup _{\substack{\omega, k \\|j|=i}}\left|\frac{\partial^{|j|} f_{k}(\omega)}{\partial \omega^{j}}\right|<A_{i}, \forall i=0, \ldots, r .
$$

Remark 8. $\operatorname{Max}_{i} A_{i}$ is a norm for $C^{r}\left(\ell^{1}, P_{2 n}\right)$.

Given a function $f \in C^{r}\left(\ell^{1}, P_{2 n}\right)$, we also consider

$$
f_{p h}(\psi):=f\left(\psi,|\psi|^{2}\right),
$$

conversely, we will say that $\tilde{f}: H^{s} \rightarrow \mathbb{C}$ is of class $P^{r}\left(2 n,\left\{A_{i}\right\}_{i=0}^{r}\right)$ if there exists a function $F(\psi, \omega) \in P^{r}\left(2 n,\left\{A_{i}\right\}_{i=0}^{r}\right)$ s.t. $F(\psi, \omega)_{\left.\right|_{\omega=\left\{\left|\psi_{k}\right|^{2}\right\}}}=\tilde{f}(\psi)$.

Remark 9. If $f \in P_{2 n}$ with $\||f|\|<\infty$, then $f \in P^{\infty}\left(2 n,\left\{A_{i}\right\}_{i=0}^{\infty}\right)$ with $A_{0}=\|f f\|$ and $A_{i}=0$ for any $i \geqslant 0$. For simplicity, we will write $f \in P^{\infty}(2 n,\||f|\|)$.

Remark 10. From lemma 3.1, for any $n \in \mathbb{N}$ and for any $s_{1}$ s.t. $\frac{1}{2}-\frac{1}{2 n}<s_{1}<\frac{1}{2}$, for any $r \geqslant 0$ and for any $f \in P^{r}\left(2 n,\left\{A_{i}\right\}_{i=0}^{r}\right)$, one has

$$
|f(\psi)| \leqslant A_{0} C\left(s_{1}, n\right)\|\psi\|_{H^{s_{1}}}^{2 n} .
$$

The connection of the norm of $P^{0}\left(2 n, A_{0}\right)$ and the $L^{2}$-norm is given by

Lemma 3.2. Let $n$ be an integer, denote $C_{g}(n):=2^{n+2}[(2 n) !]^{\frac{3}{2}}(2 n-1)^{2}\left(\sum_{l} \frac{1}{1+l^{2}}\right){ }^{n}$, then for any $\beta>0$, and $f_{p h} \in P^{0}\left(2 n, A_{0}\right)$, one has

$$
\left\|f_{p h}\right\|_{g, \beta} \leqslant \frac{A_{0} C_{g}(n)}{\beta^{n}} .
$$

Proof. Writing $f_{p h}=\sum_{k=\left(k_{1}, \ldots, k_{2 n}\right)} f_{k}(\psi) \prod_{i=1}^{n} \psi_{k_{i}} \bar{\psi}_{k_{n+i}}$, one has

$$
\left\|f_{p h}\right\|_{g, \beta}^{2}=\int_{H^{s}}\left|f_{p h}\right|^{2} \mathrm{~d} \mu_{g, \beta}=\int_{H^{s}} \sum_{k, j} f_{k}(\psi) \bar{f}_{j}(\psi) \prod_{i=1}^{n} \psi_{k_{i}} \psi_{j_{n+i}} \bar{\psi}_{j i} \bar{\psi}_{k_{n+i}} \mathrm{~d} \mu_{g, \beta} .
$$


Let $s_{1}$ be s.t. $\max \left\{s, \frac{n-1}{2 n}\right\}<s_{1}<\frac{1}{2}$, by lemma 3.1 , there exists a constant $C$ s.t. $|f|^{2} \leqslant C A_{0}^{2}\|\psi\|_{H^{s} 1}^{4 n}$, moreover by lemma $2.3,\|\psi\|_{H^{s} 1}^{4 n} \in L^{1}\left(H^{s}, \mu_{g, \beta}\right)$. So we can exchange the order between the integral and the series and (15) becomes

$$
\begin{aligned}
& \sum_{k, j} \int_{H^{s}} f_{k}(\psi) \bar{f}_{j}(\psi) \prod_{i=1}^{n} \psi_{k_{i}} \psi_{j_{n+i}} \bar{\psi}_{j_{i}} \bar{\psi}_{k_{n+i}} \mathrm{~d} \mu_{g, \beta} \\
& =\sum_{k, j} \frac{\int_{H^{s}} f_{k}(\psi) \bar{f}_{j}(\psi) \prod_{i=1}^{n} \psi_{k_{i}} \psi_{j_{n+i}} \bar{\psi}_{j i} \bar{\psi}_{k_{n+i}} \mathrm{e}^{-\frac{\beta}{2} \sum_{S_{k j}}\left(1+l^{2}\right)\left|\psi_{l}\right|^{2}} \prod_{S_{k j}} \mathrm{~d} \psi_{l} \mathrm{~d} \bar{\psi}_{l}}{\prod_{S_{k j}} \int_{H^{s}} \mathrm{e}^{-\frac{\beta}{2}\left(1+l^{2}\right)\left|\psi_{l}\right|^{2}} \mathrm{~d} \psi_{l} \mathrm{~d} \bar{\psi}_{l}}
\end{aligned}
$$

where $S_{k j}:=\operatorname{Supp}(k, j)$. It is useful to use the following notation: given a set $K$ of indices $\left(k_{1}, \ldots, k_{2 n}\right)$ with an even number of components, we denote

$$
K_{1}:=\left\{k_{1}, \ldots, k_{n}\right\}, \quad K_{2}:=\left\{k_{n+1}, \ldots, k_{2 n}\right\} .
$$

Using the substitution $\psi_{l}=\frac{\sqrt{2 z_{l}}}{\sqrt{\beta\left(1+l^{2}\right)}} \mathrm{e}^{\mathrm{i} \theta_{l}}, z_{l} \in \mathbb{R}^{+}, \theta_{l} \in[0,2 \pi)$, one has that the only integrals different from 0 are the terms in which $K_{1} \cup J_{2}=K_{2} \cup J_{1}$.

We denote by $\mathcal{T}$ the set of $(k, j)$ s.t. $K_{1} \cup J_{2}=K_{2} \cup J_{1}$ and with both $k$ and $j$ fulfilling the zero momentum condition, namely $\sum_{i=1}^{n} k_{i}=\sum_{i=n+1}^{2 n} k_{i}, \sum_{i=1}^{n} j_{i}=\sum_{i=n+1}^{2 n} j_{i}$. Thus (16) is bounded by

$$
\begin{aligned}
& A_{0}^{2} \sum_{k, j \in \mathcal{T}} \frac{2^{2 n}}{\beta^{2 n} \prod_{i=1}^{n}\left(1+k_{i}^{2}\right)\left(1+j_{n+i}^{2}\right)} \int \prod_{i=1}^{n} z_{k_{i}} z_{j i+n} \mathrm{e}^{-\sum_{S_{k j}} z_{l}} \prod_{S_{k j}} \mathrm{~d} z_{l} \\
& \leqslant A_{0}^{2} \frac{2^{2 n}(2 n) !}{\beta^{2 n}} \sum_{k, j \in \mathcal{T}} \frac{1}{\prod_{i=1}^{n}\left(1+k_{i}^{2}\right)\left(1+j_{n+i}^{2}\right)} .
\end{aligned}
$$

So,

$$
\left\|f_{p h}\right\|_{g, \beta}^{2} \leqslant \frac{A_{0}^{2} 2^{2 n}(2 n) !}{\beta^{2 n}} \sum_{(k, j) \in \mathcal{T}} \frac{1}{\prod_{i=1}^{n}\left(1+k_{i}^{2}\right)\left(1+j_{n+i}^{2}\right)} .
$$

Since we sum on $(k, j) \in \mathcal{T}$, we have that, having fixed $K_{1} \cup J_{2}=K_{2} \cup J_{1}$ we have $(2 n)$ ! way to rearrange $K_{1} \cup J_{2}$ and $(2 n)$ ! way to rearrange $K_{2} \cup J_{1}$, so

$$
\begin{aligned}
\sum_{(k, j) \in \mathcal{T}} & \frac{1}{\prod_{i=1}^{n}\left(1+k_{i}^{2}\right)\left(1+j_{n+i}^{2}\right)} \leqslant[(2 n) !]^{2} \sum_{\substack{k_{1}, \ldots, k n \\
j_{n+1}, \cdots, j_{2 n}}} \frac{1}{\prod_{i=1}^{n}\left(1+k_{i}^{2}\right)\left(1+j_{n+i}^{2}\right)} \\
& =[(2 n) !]^{2}\left(\sum_{l} \frac{1}{1+l^{2}}\right)^{2 n} .
\end{aligned}
$$

So, finally, 


$$
\left\|f_{p h}\right\|_{g, \beta}^{2} \leqslant \frac{A_{0}^{2} 2^{2 n}[(2 n) !]^{3}\left(\sum_{i} \frac{1}{1+i^{2}}\right)^{2 n}}{\beta^{2 n}} \leqslant \frac{A_{0}^{2} C_{g}^{2}(n)}{\beta^{2 n}}
$$

with $C_{g}(n)^{2}:=2^{2 n+4}[(2 n) !]^{3}(2 n-1)^{4}\left(\sum_{l} \frac{1}{1+l^{2}}\right){ }^{2 n}$.

Remark 11. According to lemma 2.1, one also has

$$
\left\|f_{p h}\right\|_{\mu_{\beta}} \leqslant \frac{A_{0} C_{g}(n)}{\beta^{n}} \text {. }
$$

The Poisson brackets of two functions $f, g$ with $f \in P_{2 n}$ and $g \in P^{r}\left(2 m,\left\{A_{i}\right\}_{i=0}^{r}\right)$ is formally, given by

$$
\{f, g\}:=L_{f}(g):=-\mathrm{i} \sum_{k}\left(\frac{\partial f}{\partial \psi_{k}} \frac{\partial g}{\partial \bar{\psi}_{k}}-\frac{\partial g}{\partial \psi_{k}} \frac{\partial f}{\partial \bar{\psi}_{k}}\right) .
$$

Remark 12. If $f \in P_{n}, g \in P_{m}$, then

$$
\{f, g\} \in P_{n+m-2} \text {. }
$$

Lemma 3.3. Consider $f \in P_{2 n},\left\|f|\||<D, g_{p h} \in P^{r}\left(2 m,\left\{A_{i}\right\}_{i=0}^{r}\right)\right.$. Then

$$
\left\{f, g_{p h}\right\}=F_{1}+F_{2} \text {, }
$$

where

$$
\begin{aligned}
& F_{1} \in P^{r}\left(2 n+2 m-2,2 n m D\left\{A_{i}\right\}_{i=0}^{r}\right), \\
& F_{2} \in P^{r-1}\left(2 n+2 m, 2 n D\left\{A_{i+1}\right\}_{i=0}^{r-1}\right) .
\end{aligned}
$$

Proof. Writing $g_{p h}=\sum_{k=\left(k_{1}, \ldots k_{2 m}\right)} g_{k}\left(\left\{\left|\psi_{k}\right|^{2}\right\}\right) \psi_{k_{1}} \ldots \psi_{k_{m}} \bar{\psi}_{k_{m+1}} \ldots \bar{\psi}_{k_{2 m}}$, then it is immediate to verify that (19) holds with

$$
\begin{aligned}
& F_{1}=\sum_{k=\left(k_{1}, \ldots k_{2 m}\right)} g_{k}\left(\left\{\left|\psi_{j}\right|^{2}\right\}\right)\left\{f, \psi_{k_{1}} \ldots \psi_{k_{m}} \bar{\psi}_{k_{m+1}} \ldots \bar{\psi}_{k_{2 m}}\right\} \\
& F_{2}=\sum_{k=\left(k_{1}, \ldots k_{2 m}\right)} \psi_{k_{1} \ldots \psi_{k_{m}}} \bar{\psi}_{k_{m+1}} \ldots \bar{\psi}_{k_{2 m}}\left\{f, g_{k}\left(\left\{\left|\psi_{j}\right|^{2}\right\}\right)\right\} \\
& =\sum_{k=\left(k_{1}, \ldots k_{2 m}\right)}\left(\sum_{l} \frac{\partial g_{k}\left(\left\{\left|\psi_{j}\right|^{2}\right\}\right)}{\partial \omega_{l}}\right) \psi_{k_{1} \ldots} \ldots \psi_{k_{m}} \bar{\psi}_{k_{m+1}} \ldots \bar{\psi}_{k_{2 m}}\left\{f,\left|\psi_{l}\right|^{2}\right\}
\end{aligned}
$$

and, by remark $12, \quad F_{1} \in P^{r}\left(2 n+2 m-2,2 n m D\left\{A_{i}\right\}_{i=0}^{r}\right) \quad$ and $\quad F_{2} \in P^{r-1}(2 n+$ $\left.2 m, 2 n D\left\{A_{i+1}\right\}_{i=0}^{r-1}\right)$ hold.

Actually, we shall use a more particular class of functions in which the range of the indices is subject to a further restriction. This is related to the fact that in our construction we shall 
fix an index $\mathrm{k}$ corresponding to the action we want to conserve. To this end, we introduce the following definition:

Definition 4. Given $M>0, \mathrm{k} \in \mathbb{Z}$, a linear combination

$$
G\left(k_{1}, \ldots, k_{2 n}\right):=\sum_{i=1}^{2 n} a_{i} k_{i}
$$

with $a_{i} \in \mathbb{Z},\left|a_{i}\right| \leqslant M$, we will say that the relation

$$
G\left(k_{1}, \ldots, k_{2 n}\right)=\mathrm{k}
$$

is $(M, \mathrm{k})$-admissible.

Lemma 3.4. Given $D>0$, let be $f \in P_{2 n}$, $\|f\| \|<D, g_{p h}(\psi, \bar{\psi}) \in P^{r}\left(2 m,\left\{A_{i}\right\}_{i=0}^{r}\right), M>0$, $\mathrm{k} \in \mathbb{Z}$.

Assume that

$$
g_{p h}=\sum_{\substack{k=\left(k_{1}, \ldots, k_{2 m}\right) \text { s.t. } \\ G_{k}\left(k_{1}, \ldots, k_{2 m}\right)=\mathrm{k}}} g_{k}\left(\left\{\left|\psi_{k}\right|^{2}\right\}\right) \psi_{k_{1}} \ldots \psi_{k_{m}} \bar{\psi}_{k_{m+1} \ldots} \bar{\psi}_{k_{2 m}}
$$

where, for any $k, G_{k}=\mathrm{k}$ is $(M, \mathrm{k})$-admissible. Then

$$
\left\{f, g_{p h}\right\}=F_{1}+F_{2}
$$

where

$$
\begin{aligned}
& F_{1}=\sum_{\substack{k^{\prime}=\left(k_{1}^{\prime}, \ldots, k_{2 n+2 m-2}^{\prime}\right) \\
\tilde{G}_{k^{\prime}}\left(k_{1}^{\prime} \ldots, k_{2 n+2 m-2}^{\prime}\right)=\mathrm{k}}} F_{1, k^{\prime}} \psi_{k_{1}^{\prime}} \ldots \psi_{k_{n+m-1}^{\prime}} \bar{\psi}_{k_{n+m}^{\prime} \ldots} \bar{\psi}_{k_{2 m+2 n-2}^{\prime}} \\
& F_{2}=\sum_{\substack{k^{\prime \prime}=\left(k_{1}^{\prime \prime}, \ldots, k_{2 n+2 m}^{\prime \prime}\right) \\
\hat{G}_{k^{\prime \prime}}\left(k_{1}^{\prime \prime}, \ldots, k_{2 n+2 m}^{\prime \prime}\right)=\mathrm{k}}} F_{2, k^{\prime \prime}} \psi_{k_{1}^{\prime \prime} \ldots} \ldots \psi_{k_{m+n}^{\prime \prime}} \bar{\psi}_{k_{m+n+1}^{\prime \prime}} \ldots \bar{\psi}_{k_{2 m+2 n}^{\prime \prime}}
\end{aligned}
$$

where for any $k^{\prime}, k^{\prime \prime}$, the relations $\tilde{G}_{k^{\prime}}=\mathrm{k}, \hat{G}_{k^{\prime \prime}}=\mathrm{k}$ are $(2 M, \mathrm{k})$-admissible.

Proof. Writing $f=\sum_{l=\left(l_{1}, \ldots l_{2 n}\right)} f_{l} \psi_{l_{1} \ldots} \ldots \psi_{l_{n}} \bar{\psi}_{l_{n+1} \ldots} \bar{\psi}_{l_{2 n}}$, by lemma 3.3 , we have $F_{1} \in P^{r}\left(2 n+2 m-2,2 n m D\left\{A_{i}\right\}_{i=0}^{r}\right), F_{2} \in P^{r-1}\left(2 n+2 m, 2 n D\left\{A_{i}\right\}_{i=1}^{r}\right)$. Moreover, each term of $F_{1}$ is originated by two terms that depend respectively on $l=\left(l_{1}, \ldots l_{2 n}\right)$ and $k=\left(k_{1}, \ldots k_{2 m}\right)$ s.t. $\sum_{i=1}^{n} l_{i}=\sum_{i=n+1}^{2 n} l_{i}, \sum_{i=1}^{m} k_{i}=\sum_{i=m+1}^{2 m} k_{i}$ and $\left\{l_{1}, \ldots l_{n}\right\} \cap\left\{k_{m+1}, \ldots k_{2 m}\right\} \neq \varnothing$ or $\left\{l_{n+1}, \ldots l_{2 n}\right\} \cap\left\{k_{1}, \ldots k_{m}\right\} \neq \varnothing$. Without loosing generality, we can suppose $l_{1}=k_{m+1}$.

We form a vector of indices $k^{\prime}=\left(l_{2}, \ldots l_{n}, k_{1}, \ldots, k_{m}, l_{n+1}, \ldots l_{2 n}, k_{m+2}, \ldots, k_{2 m}\right) \quad$ s.t. $\sum_{i=2}^{n} l_{i}+\sum_{i=1}^{m} k_{i}=\sum_{i=n+1}^{2 n} k_{i}+\sum_{i=m+2}^{2 m} k_{i}$. Moreover, $k_{m+1}=\sum_{i=1}^{m} k_{i}-\sum_{i=m+2}^{2 m} k_{i}$. Ву hypothesis, we can write $G_{k}\left(k_{1}, \ldots, k_{2 m}\right)=\sum_{i=1}^{2 m} a_{i} k_{i}$ with $a_{i} \in \mathbb{N},\left|a_{i}\right|<M$, so

$$
\mathrm{k}=G_{k}\left(k_{1}, \ldots, k_{2 m}\right)=\sum_{i=1}^{2 m} a_{i} k_{i}=\sum_{i=1}^{m}\left(a_{i}+a_{m+1}\right) k_{i}+\sum_{i=m+2}^{2 m}\left(a_{i}-a_{m+1}\right) k_{i}
$$




$$
\begin{aligned}
& =\sum_{i=1}^{m} b_{i} k_{i}+\sum_{i=m+2}^{2 m} b_{i} k_{i}=\tilde{G}_{k}\left(k_{1}, \ldots, k_{m}, k_{m+2}, \ldots, k_{2 m}\right) \\
& =\tilde{G}_{k^{\prime}}\left(l_{2}, \ldots, l_{n}, k_{1}, \ldots, k_{m}, l_{n+1}, \ldots, l_{2 n}, k_{m+2}, \ldots, k_{2 m}\right) .
\end{aligned}
$$

We note that $\left|b_{i}\right|<2 M$ and $\tilde{G}_{k}$ is a linear combination only of $\left\{k_{1}, \ldots, k_{m}, k_{m+2}, \ldots, k_{2 m}\right\}$ so it is independent of the null-momentum condition related to $\left(l_{2}, \ldots, l_{n}, k_{1}, \ldots, k_{m}, l_{n+1}, \ldots, l_{2 n}, k_{m+2}, \ldots, k_{2 m}\right)$, so we obtain the thesis for $F_{1}$. For $F_{2}$ the situation is simpler. Again each term of $F_{2}$ is originated by two terms that depend respectively on $l$ and $k$ s.t. $\sum_{i=1}^{n} l_{i}=\sum_{i=n+1}^{2 n} l_{i}, \sum_{i=1}^{m} k_{i}=\sum_{i=m+1}^{2 m} k_{i}$ and $\left\{l_{1}, \ldots l_{n}\right\} \cap\left\{k_{m+1}, \ldots k_{2 m}\right\} \neq \emptyset$ or $\left\{l_{n+1}, \ldots l_{2 n}\right\} \cap\left\{k_{1}, \ldots k_{m}\right\} \neq \emptyset$.

We obtain a vector of indices $k^{\prime \prime}=\left(l_{1}, \ldots, l_{n}, k_{1}, \ldots, k_{m}, l_{n+1}, \ldots, l_{2 n}, k_{m+1}, \ldots, k_{2 m}\right)$ s.t. $\sum_{i=1}^{n} l_{i}+\sum_{i=1}^{m} k_{i}=\sum_{i=n+1}^{2 n} k_{i}+\sum_{i=m+1}^{2 m} k_{i}$ and

$$
\mathrm{k}=G_{k}\left(k_{1}, \ldots k_{2 m}\right)=\tilde{G}_{k^{\prime \prime}}\left(l_{1}, \ldots, l_{n}, k_{1}, \ldots, k_{m}, l_{n+1}, \ldots, l_{2 n}, k_{m+1}, \ldots, k_{2 m}\right) .
$$

Remark 13. This result holds also in the particular case in which $g_{k}$ is a constant independent of $\left\{\left|\psi_{j}\right|^{2}\right\}$.

In particular, one can obtain the following improvement of lemma 3.2:

Lemma 3.5. Let $n$ be an integer, $M>0, \mathrm{k} \in \mathbb{Z}$, let

$$
P^{0}\left(2 n, A_{0}\right) \ni f_{p h}=\sum_{\substack{k=\left(k_{1}, \ldots, k_{2 n}\right) \\ G_{k}\left(k_{1}, \ldots, k_{2 n}\right)=\mathrm{k}}} f_{k}\left(\left\{\left|\psi_{k}\right|^{2}\right\}\right) \psi_{k_{1}} \ldots \psi_{k_{n}} \bar{\psi}_{k_{n+1} \ldots} \bar{\psi}_{k_{2 n}},
$$

and assume that, for any $k, G_{k}\left(k_{1}, \ldots, k_{2 n}\right)=\mathrm{k}$ is $(M, \mathrm{k})$-admissible.

Then, for any $\beta>0$, one has

$$
\left\|f_{p h}\right\|_{g, \beta} \leqslant \frac{A_{0} C_{g}(n) M^{2}}{\left(1+\mathrm{k}^{2}\right) \beta^{n}}
$$

The proof of this lemma is very technical and it is deferred to appendix B.1.

\section{Formal construction of perturbed actions}

In this section we look for a formal integral of motion which is a higher order perturbation of $\Phi_{\mathrm{k}, 2}:=\left|\psi_{\mathrm{k}}\right|^{2}$. Thus we fix once for all the value of $\mathrm{k}$.

To present the construction, we describe first an equivalent one, which however is difficult to manage directly. Since $\mathrm{H}_{2}$ is completely resonant, it is well known that one can construct, formally a canonical transformation $T$ which transforms the Hamiltonian into

$$
H_{2}+Z_{4}+Z_{6}+R_{8}
$$

with $Z_{4}$ and $Z_{6}$ which Poisson commute with $H_{2}$. In particular $Z_{4}$ has been computed in many papers (see e.g. [1]) and is given by

$$
Z_{4}(\psi):=\frac{c_{2}}{2}\left(\sum_{k}\left|\psi_{k}\right|^{2}\right)^{2}-\frac{c_{2}}{2} \sum_{k}\left|\psi_{k}\right|^{4}
$$


Then, following the ideas by Poincaré, we look for $\tilde{\Phi}_{\mathrm{k}, 6}$, Poisson commuting with $\mathrm{H}_{2}$, s.t. $\tilde{\Phi}_{\mathrm{k}}^{(6)}:=\Phi_{\mathrm{k}, 2}+\tilde{\Phi}_{\mathrm{k}, 6}$ is an approximate integral of motion of (25). Computing the Poisson bracket of this quantity with (25), one has that this is a quantity of order at least 8 if

$$
\left\{Z_{4}, \tilde{\Phi}_{\mathrm{k}, 6}\right\}=\left\{\Phi_{\mathrm{k}, 2}, Z_{6}\right\}=: \mathcal{R}_{6},
$$

which is clearly impossible since the lhs is of order 8 and the rhs of order 6 , so we will modify it. Since $Z_{4}$ depends on the actions only, one has

$$
\left\{Z_{4}, \cdot\right\}=\mathrm{i} \sum_{j} \omega_{j}\left(\psi_{j} \frac{\partial}{\partial \psi_{j}}-\bar{\psi}_{j} \frac{\partial}{\partial \bar{\psi}_{j}}\right),
$$

with $\omega_{j}:=c_{2}\left(\left|\psi_{j}\right|^{2}+\sum_{k}\left|\psi_{k}\right|^{2}\right)$. So one is led to separate the regions where the $\omega_{j}$ 's are resonant and those in which they are non resonant. The resonant regions and the nonresonant regions will be defined precisely in the following. Denote $\mathcal{R}_{6}^{\mathrm{NR}}$ the restriction of $Z_{6}$ to the nonresonant regions, we will solve the equation

$$
\left\{Z_{4}, \tilde{\Phi}_{\mathrm{k}, 6}\right\}=\mathcal{R}_{6}^{\mathrm{NR}}
$$

Looking for $\tilde{\Phi}_{\mathrm{k}}^{(6)}$ in the class of polynomials with frequency dependent coefficients, the approximate integral of motion that we are going to construct is given by the sixth order truncation of $T^{-1} \tilde{\Phi}_{\mathrm{k}}^{(6)}$. We proceed now to the construction of the integral of motion. Define the operator $L_{H_{2}}:=\left\{H_{2}, \cdot\right\}$, we have that for any $f \in P_{2 n}$

$$
L_{\mathrm{H}_{2}} f=\left\{H_{2}, f\right\} \equiv-\mathrm{i} \sum_{l, m} f_{l, m}\left\langle\mathbf{k}^{2},(l-m)\right\rangle \psi^{l} \bar{\psi}^{m}
$$

where $\left\langle\mathbf{k}^{2},(l-m)\right\rangle:=\sum_{j} k_{j}^{2}\left(l_{j}-m_{j}\right)$.

Equivalently, for any $f \in P_{2 n}$, we can write

$$
L_{H_{2}} f=-\mathrm{i} \sum_{k} f_{k}\left(\sum_{k} k^{2}\left(\sum_{i=1}^{n} \delta_{k_{i}, k}-\sum_{i=n+1}^{2 n} \delta_{k_{i}, k}\right)\right) \prod_{i=1}^{n} \psi_{k_{i}} \bar{\psi}_{k_{i+n}},
$$

where $\delta_{x, y}$ is kronecker's delta.

Definition 5. We denote by

$$
\begin{aligned}
& N_{H_{2}}:=\operatorname{ker} L_{H_{2}}=\left\{f \in \cup_{n \in \mathbb{N}} P_{2 n}: f_{l, m} \neq 0 \Leftrightarrow\left\langle\mathbf{k}^{2},(l-m)\right\rangle=0\right\}, \\
& R_{H_{2}}:=\left\{f \in \cup_{n \in \mathbb{N}} P_{2 n}: f_{l, m} \neq 0 \Leftrightarrow\left\langle\mathbf{k}^{\mathbf{2}},(l-m)\right\rangle \neq 0\right\} .
\end{aligned}
$$

Remark 14. $L_{H_{2}}: R_{H_{2}} \rightarrow R_{H_{2}}$ is formally invertible.

Given a polynomial $f$, we indicate the projection of $f$ on $N_{H_{2}}$ by $f^{N_{H_{2}}}$ and the projection on $R_{\mathrm{H}_{2}}$ by $f^{R_{H_{2}}}$.

In particular, we have

$$
H_{4}^{R_{H_{2}}}:=\frac{c_{2}}{4} \sum_{\substack{k_{1}+k_{2}=k_{3}+k_{4} \\ k_{1}^{2}+k_{2}^{2} \neq k_{3}^{2}+k_{4}^{2}}} \psi_{k_{1}} \psi_{k_{2}} \bar{\psi}_{k_{3}} \bar{\psi}_{k_{4}}
$$




$$
Z_{4}=H_{4}^{N_{H_{2}}}
$$

Define now

$$
\begin{aligned}
& \chi_{4}:=-L_{H_{2}}^{-1} H_{4}^{R_{H_{2}}}, \chi_{6}:=-L_{H_{2}}^{-1}\left(\frac{1}{2}\left\{\chi_{4}, H_{4}^{R_{H_{2}}}\right\}+\left\{\chi_{4}, Z_{4}\right\}+H_{6}\right)^{R_{H_{2}}}, \\
& \Phi_{k, 4}:=L_{\chi_{4}}\left|\psi_{k}\right|^{2}, \Phi_{k, 6}:=\frac{1}{2} L_{\chi_{4}}^{2}\left|\psi_{k}\right|^{2}+L_{\chi_{6}}\left|\psi_{k}\right|^{2}
\end{aligned}
$$

and

$$
Z_{6}:=H_{6}^{N_{H_{2}}}+\left(\frac{1}{2}\left\{\chi_{4}, H_{4}^{R_{H_{2}}}\right\}+\left\{\chi_{4}, Z_{4}\right\}\right)^{N_{H_{2}}},
$$

to proceed, we have to define the resonant/nonresonant decomposition of the phase-space.

Definition 6. For any $n>0$, we denote by

$$
\mathcal{M}_{2 n}:=\left\{k=\left\{k_{j}\right\} \in \mathbb{Z}^{2 n} \text { s.t } \sum_{j=1}^{n} k_{j}=\sum_{j=n+1}^{2 n} k_{j}, \sum_{j=1}^{n} k_{j}^{2}=\sum_{j=n+1}^{2 n} k_{j}^{2}\right\} .
$$

Write

$$
Z_{6}=\sum_{k \in \mathcal{M}_{6}} \tilde{Z}_{6, k} \psi_{k_{1}} \psi_{k_{2}} \psi_{k_{3}} \bar{\psi}_{k_{4}} \bar{\psi}_{k_{5}} \bar{\psi}_{k_{6}}
$$

computing

$$
\mathcal{R}_{6}=\left\{\Phi_{\mathrm{k}, 2}, Z_{6}\right\},
$$

one gets

$$
\mathcal{R}_{6}=\sum_{k \in \mathcal{M}_{6}} Z_{6, k, \mathrm{k}}
$$

with

$$
Z_{6, k, \mathrm{k}}:=-\mathrm{i} \tilde{Z}_{6, k}\left(\delta_{k_{1}, \mathrm{k}}+\delta_{k_{2}, \mathrm{k}}+\delta_{k_{3}, \mathrm{k}}-\delta_{k_{4}, \mathrm{k}}-\delta_{k_{5}, \mathrm{k}}-\delta_{k_{6}, \mathrm{k}}\right) \psi_{k_{1}} \psi_{k_{2}} \psi_{k_{3}} \bar{\psi}_{k_{4}} \bar{\psi}_{k_{5}} \bar{\psi}_{k_{6}},
$$

where $\delta_{j, \mathrm{k}}$ is Kronecker's delta.

We introduce a function $\rho \in \mathcal{C}_{0}^{\infty}$, s.t.

$$
\rho(x)=\left\{\begin{array}{l}
1 \text { if }|x|>2 \\
0 \text { if }|x|<1
\end{array} .\right.
$$

Recalling that $\omega_{j}:=c_{2}\left(\left|\psi_{j}\right|^{2}+\sum_{k}\left|\psi_{k}\right|^{2}\right)$, we denote by

$$
\begin{aligned}
a_{k}(\psi) & :=\frac{1}{c_{2}}\left(\omega_{k_{1}}+\omega_{k_{2}}+\omega_{k_{3}}-\omega_{k_{4}}-\omega_{k_{5}}-\omega_{k_{6}}\right) \\
& =\left(\left|\psi_{k_{1}}\right|^{2}+\left|\psi_{k_{2}}\right|^{2}+\left|\psi_{k_{3}}\right|^{2}-\left|\psi_{k_{4}}\right|^{2}-\left|\psi_{k_{5}}\right|^{2}-\left|\psi_{k_{6}}\right|^{2}\right)
\end{aligned}
$$

and, given $0<\delta<1$, we define the decomposition $\mathcal{R}_{6}:=\mathcal{R}_{6}^{\mathrm{NR}}+\mathcal{R}_{6}^{R}$ with

$$
\mathcal{R}_{6}^{\mathrm{NR}}:=\sum_{k} Z_{6, k, \mathrm{k}} \rho\left(\frac{a_{k}(\psi)}{\delta}\right)
$$


and

$$
\mathcal{R}_{6}^{R}:=\sum_{k} Z_{6, k, \mathrm{k}}\left(1-\rho\left(\frac{a_{k}(\psi)}{\delta}\right)\right) .
$$

We define $\tilde{\Phi}_{k, 6}$ to be the solution of equation (28), which is explicitly given by

$$
\tilde{\Phi}_{\mathrm{k}, 6}:=\mathrm{i} \sum_{k \in \mathcal{M}_{6}} \frac{Z_{6, k, \mathrm{k}}}{c_{2} a_{k}(\psi)} \rho\left(\frac{a_{k}(\psi)}{\delta}\right) .
$$

Remark 15. $\quad \tilde{\Phi}_{\mathrm{k}, 6}(\psi) \in P^{2}\left(6,\left\{\frac{A_{i}}{\delta^{i}}\right\}_{i=0}^{2}\right) \subset P^{2}\left(6,\left\{\frac{A}{\delta^{i}}\right\}_{i=0}^{2}\right)$ with $A:=\max _{i} A_{i}$.

Finally we define the approximate integral of motion is given by

$$
\Phi_{\mathrm{k}}^{(6)}:=\Phi_{\mathrm{k}, 2}+\Phi_{\mathrm{k}, 4}+\Phi_{\mathrm{k}, 6}+\tilde{\Phi}_{\mathrm{k}, 6}+L_{\chi_{4}} \tilde{\Phi}_{\mathrm{k}, 6} .
$$

The following lemma gives the structure of its time derivative.

\section{Lemma 4.1. Write}

$$
\left\{H, \Phi_{\mathrm{k}}^{(6)}\right\}=-\mathcal{R}_{6}^{R}+R
$$

then

$$
R=\sum_{j=4}^{q+1} R_{2 j}+\sum_{j=5}^{q+2} R_{2 j, 1}+\sum_{j=6}^{q+3} R_{2 j, 2}+\sum_{j=7}^{q+5} R_{2 j, 3},
$$

with $R_{2 j} \in P_{2 j}$, and there exists $C>0$ s.t.

$$
R_{2 j, l} \in P^{3-l}\left(2 j,\left\{\frac{C}{\delta^{m+l}}\right\}_{m=0}^{3-l}\right) .
$$

Proof. One has

$$
\begin{aligned}
&\left\{H, \Phi_{\mathrm{k}}^{(6)}\right\}=\left\{H_{2}, \Phi_{\mathrm{k}, 2}\right\} \\
&+\left\{H_{2}, \Phi_{\mathrm{k}, 4}\right\}+\left\{H_{4}, \Phi_{\mathrm{k}, 2}\right\}+\left\{H_{2}, \tilde{\Phi}_{\mathrm{k}, 6}\right\} \\
&+\left\{Z_{6}, \Phi_{k, 2}\right\}+\left\{Z_{4}, \tilde{\Phi}_{\mathrm{k}, 6}\right\}+\left\{H_{4}^{R_{H}}, \tilde{\Phi}_{\mathrm{k}, 6}\right\}+\left\{H_{2}, L_{\chi_{4}} \tilde{\Phi}_{\mathrm{k}, 6}\right\} \\
&+\sum_{j=2}^{n-2}\left(\left\{H_{2 j}, \Phi_{k, 6}\right\}+\left\{H_{2 j}, L_{\chi_{4}} \tilde{\Phi}_{\mathrm{k}, 6}\right\}+\left\{H_{2(j+1)}, \Phi_{k, 4}\right\}+\left\{H_{2(j+1)}, \tilde{\Phi}_{\mathrm{k}, 6}\right\}+\left\{H_{2(j+2)}, \Phi_{k, 2}\right\}\right) \\
&+\left\{H_{2(n-1)}, \Phi_{k, 6}\right\}+\left\{H_{2(n-1)}, L_{\chi_{4}} \tilde{\Phi}_{\mathrm{k}, 6}\right\}+\left\{H_{2 n}, \Phi_{k, 6}\right\}+\left\{H_{2 n}, \tilde{\Phi}_{\mathrm{k}, 6}\right\} \\
&+\left\{H_{2 n}, \Phi_{k, 6}\right\}+\left\{H_{2 n}, L_{\chi_{4}} \tilde{\Phi}_{\mathrm{k}, 6}\right\} .
\end{aligned}
$$


Due to the construction, we have that $\left\{H_{2}, \Phi_{\mathrm{k}, 2}\right\}=0$ and $\left\{H_{2}, \Phi_{\mathrm{k}, 4}\right\}=-\left\{H_{4}, \tilde{\Phi}_{\mathrm{k}, 2}\right\}$. Due to the fact that $a_{k}$ and $\rho$ depend on the actions only and $\left\{Z_{6, k, \mathrm{k}}, H_{2}\right\}=0$, one has $\left\{H_{2}, \widetilde{\Phi}_{\mathrm{k}, 6}\right\}=0$ so that (34) vanishes.

Since $Z_{4}$ is a function of the actions only, we have also

$$
\left\{Z_{4}, \tilde{\Phi}_{\mathrm{k}, 6}\right\}=\mathrm{i} \sum_{k}\left\{Z_{4}, Z_{6, k, \mathrm{k}}\right\} \frac{\rho\left(\frac{a_{k}(\psi)}{\delta}\right)}{c_{2} a_{k}(\psi)}=\sum_{k} Z_{6, k, \mathrm{k}} \rho\left(\frac{a_{k}(\psi)}{\delta}\right)=\mathcal{R}_{6}^{\mathrm{NR}} .
$$

We note that $\left\{H_{4}^{R_{H_{2}}}, \tilde{\Phi}_{\mathrm{k}, 6}\right\}=-\left\{H_{2}, L_{\chi_{4}} \tilde{\Phi}_{\mathrm{k}, 6}\right\}$ in fact, by the definition of $\chi_{4}$ and $\left\{H_{2}, \tilde{\Phi}_{\mathrm{k}, 6}\right\}=0$, one has

$$
\begin{aligned}
& \left\{H_{2}, L_{\chi_{4}} \tilde{\Phi}_{\mathrm{k}, 6}\right\}=-\left\{H_{2},\left\{L_{H_{2}}^{-1} H_{4}^{R_{H_{2}}}, \tilde{\Phi}_{\mathrm{k}, 6}\right\}\right\} \\
& =\left\{L_{H_{2}}^{-1} H_{4}^{R_{H_{2}}},\left\{\tilde{\Phi}_{\mathrm{k}, 6}, H_{2}\right\}\right\}+\left\{\tilde{\Phi}_{\mathrm{k}, 6}, L_{H_{2}} L_{H_{2}}^{-1} H_{4}^{R_{H_{2}}}\right\}=\left\{\tilde{\Phi}_{\mathrm{k}, 6}, H_{4}^{R_{H_{2}}}\right\} .
\end{aligned}
$$

So, by (29), line (35) reduces to $\sum_{k} Z_{6, k, \mathrm{k}}\left(\rho\left(\frac{a_{k}(\psi)}{\delta}\right)-1\right)=-\mathcal{R}_{6}^{R}$.

It remains to study now (36)-(38). Using lemma 3.3, we have

$$
\begin{aligned}
& \left\{H_{2 j}, \tilde{\Phi}_{\mathrm{k}, 6}\right\}=F_{1, j}+F_{2, j}, \\
& F_{1, j} \in P^{2}\left(2 j+4,\left\{\frac{C}{\delta^{i+1}}\right\}_{i=0}^{2}\right), F_{2, j} \in P^{1}\left(2 j+6,\left\{\frac{C}{\delta^{i+2}}\right\}_{i=0}^{1}\right) \\
& L_{\chi_{4}} \tilde{\Phi}_{\mathrm{k}, 6}=E_{1}+E_{2}, E_{1} \in P^{1}\left(8,\left\{\frac{C}{\delta^{i+1}}\right\}_{i=0}^{2}\right), E_{2} \in P^{2}\left(10,\left\{\frac{C}{\delta^{i+2}}\right\}_{i=0}^{1}\right),
\end{aligned}
$$

so

$$
\begin{aligned}
& \left\{H_{2 j}, L_{\chi_{4}} \tilde{\Phi}_{\mathrm{k}, 6}\right\}=F_{3, j}+F_{4, j}+F_{5, j}, \\
& F_{3, j} \in P^{2}\left(2 j+6,\left\{\frac{C}{\delta^{i+1}}\right\}_{i=0}^{2}\right), F_{4, j} \in P^{1}\left(2 j+8,\left\{\frac{C}{\delta^{i+2}}\right\}_{i=0}^{1}\right), \\
& F_{5, j} \in P^{0}\left(2 j+10, \frac{C}{\delta^{3}}\right) \\
& \left\{H_{2 j}, \Phi_{k, 2}\right\} \in P_{2 j}, \\
& \left\{H_{2 j}, \Phi_{k, 4}\right\} \in P_{2 j+2}, \\
& \left\{H_{2 j}, \Phi_{k, 6}\right\} \in P_{2 j+4} .
\end{aligned}
$$




\section{Measure estimates}

In this section we estimate $\left\|\left|\psi_{\mathrm{k}}\right|^{2}\right\|_{\mu_{\beta}},\left\|\Phi_{\mathrm{k}}^{(6)}\right\|_{\mu_{\beta}}$ and $\left\|\left\{H, \Phi_{\mathrm{k}}^{(6)}\right\}\right\|_{\mu_{\beta}}$.

Lemma 5.1. There exists a constant $C>0$ s.t. for any $\beta>1, \delta \in(0,1)$ s.t. $0<\delta \beta<1$, one has

$$
\begin{aligned}
\left\|\Phi_{\mathrm{k}}^{(6)}-\left|\psi_{\mathrm{k}}\right|^{2}\right\|_{g, \beta}^{2} & \leqslant \frac{C}{\left(1+\mathrm{k}^{2}\right)^{2} \min \left\{\delta^{2} \beta^{6}, \delta^{4} \beta^{10}\right\}}, \\
\|R\|_{g, \beta}^{2} & \leqslant \frac{C}{\left(1+\mathrm{k}^{2}\right)^{2} \delta^{6} \beta^{14}},
\end{aligned}
$$

where $R$ is defined by (33).

Proof. We recall that

$$
\Phi_{\mathrm{k}}^{(6)}-\left|\psi_{\mathrm{k}}\right|^{2}=\Phi_{\mathrm{k}, 4}+\Phi_{\mathrm{k}, 6}+\tilde{\Phi}_{\mathrm{k}, 6}+L_{\chi_{4}} \tilde{\Phi}_{\mathrm{k}, 6} .
$$

By construction, $\Phi_{\mathrm{k}, 4} \in P_{4}, \quad \Phi_{\mathrm{k}, 6} \in P_{6} \quad$ and there exists $\quad C_{1}>0 \quad$ s.t. $\Phi_{\mathrm{k}, 6} \in P^{2}\left(6,\left\{\frac{C_{1}}{\delta^{i+1}}\right\}_{i=0}^{2}\right)$ and, using lemma 3.4 , there exists $C_{2}>0$ s.t. $L_{\chi_{4}} \tilde{\Phi}_{\mathrm{k}, 6}=E_{1}+E_{2}, E_{1} \in P^{2}\left(8,\left\{\frac{C_{2}}{\delta^{i+1}}\right\}_{i=0}^{2}\right), E_{2} \in P^{1}\left(10,\left\{\frac{C_{2}}{\delta^{i+2}}\right\}_{i=0}^{1}\right)$.

Moreover, $\quad P^{2}\left(6,\left\{\frac{C_{1}}{\delta^{i+1}}\right\}_{i=0}^{2}\right) \subset P^{0}\left(6, \frac{C_{1}}{\delta}\right), \quad P^{2}\left(8,\left\{\frac{C_{2}}{\delta^{i+1}}\right\}_{i=0}^{2}\right) \subset P^{0}\left(8, \frac{C_{2}}{\delta}\right) \quad$ and $P^{1}\left(10,\left\{\frac{C_{2}}{\delta^{i+2}}\right\}_{i=0}^{1}\right) \subset P^{0}\left(10, \frac{C_{2}}{\delta^{2}}\right)$. So, using lemmas 3.4 and 3.5 with $M=2$, we obtain

$$
\begin{aligned}
\left\|\Phi_{\mathrm{k}}^{(6)}-\left|\psi_{\mathrm{k}}\right|^{2}\right\|_{g, \beta}^{2} & \leqslant \frac{C}{\left(1+\mathrm{k}^{2}\right)^{2}}\left(\frac{1}{\beta^{4}}+\frac{1}{\beta^{6}}+\frac{1}{\delta^{2} \beta^{6}}+\frac{1}{\delta^{2} \beta^{8}}+\frac{1}{\delta^{4} \beta^{10}}\right) \\
& \leqslant \frac{5 C}{\left(1+\mathrm{k}^{2}\right)^{2} \delta^{2} \beta^{6}}
\end{aligned}
$$

where we used $0<\delta \beta<1$. Using (33), lemmas 4.1, 3.4 and 3.5 with $M=4$, we get

$$
\|R\|_{g, \beta}^{2} \leqslant \frac{C}{\left(1+\mathrm{k}^{2}\right)^{2}}\left(\sum_{j=4}^{n+1} \frac{1}{\beta^{2 j}}+\sum_{j=5}^{n+2} \frac{1}{\delta^{2} \beta^{2 j}}+\sum_{j=6}^{n+3} \frac{1}{\delta^{4} \beta^{2 j}}+\sum_{j=7}^{n+5} \frac{1}{\delta^{6} \beta^{2 j}}\right)
$$

so

$$
\|R\|_{g, \beta}^{2} \leqslant \frac{C}{\left(1+\mathrm{k}^{2}\right)^{2} \min \left\{\delta^{2} \beta^{6}, \delta^{4} \beta^{10}\right\}} .
$$

It remains to estimate the resonant part, namely $\left\|\mathcal{R}_{6}^{R}\right\|_{g, \beta}^{2}$.

Lemma 5.2. There exists a constant $\tilde{C}>0$ s.t. for any $\beta>0$ and $\delta>0$ s.t. $0<\delta \beta<1$, one has

$$
\left\|\mathcal{R}_{6}^{R}\right\|_{g, \beta}^{2} \leqslant \tilde{C} \frac{(\delta \beta)^{\frac{2}{3}}}{\beta^{6}\left(1+\mathrm{k}^{2}\right)^{2}} .
$$


The very technical proof is deferred to the appendix B. We remark that the difficult part consists in showing the presence of $\left(1+\mathrm{k}^{2}\right)^{2}$ at the denominators.

Finally, we obtain the following

Lemma 5.3. There exists a constant $C>0$ s.t. for any $\beta>0$, one has

$$
\left\|\dot{\Phi}_{\mathrm{k}}^{(6)}\right\|_{g, \beta}=\left\|\left\{H, \Phi_{\mathrm{k}}^{(6)}\right\}\right\|_{g, \beta} \leqslant \frac{C}{\left(1+\mathrm{k}^{2}\right) \beta^{3+\frac{1}{10}}} .
$$

Proof. By lemma 4.1, we know that

$$
\left\{H, \Phi_{\mathrm{k}}^{(6)}\right\}=-\mathcal{R}_{6}^{R}+R .
$$

Using lemmas 5.1 and 5.3, we can choose $\delta$ in such a way that (40) and (41) have the same size:

$$
\frac{1}{\delta^{6} \beta^{14}}=\frac{(\delta \beta)^{\frac{2}{3}}}{\beta^{6}} .
$$

It follows that $\delta=\frac{1}{\beta^{\frac{13}{10}}}$ and the thesis.

Finally, using these results and lemma 2.1, we obtain

Lemma 5.4. There exists $\beta^{*}, C>0$ s.t. for any $\beta>\beta^{*}$, one has

$$
\left\|\dot{\Phi}_{\mathrm{k}}^{(6)}\right\|_{\mu_{\beta}} \leqslant \frac{C}{\left(1+\mathrm{k}^{2}\right) \beta^{3+\frac{1}{10}}} .
$$

Proof. This results is a simple consequence of lemmas 2.1 and 5.3.

\section{Proof of theorem 1.1}

Proof of theorem 1.1. Using Chebyshev's inequality, one has

$\mu_{\beta}\left\{\psi:\left|\Phi_{\mathrm{k}}^{(6)}(\psi(t))-\Phi_{\mathrm{k}}^{(6)}(\psi(0))\right|>\eta_{1}\left\|\left.|| \psi_{\mathrm{k}}\right|^{2}\right\|_{\mu_{\beta}}\right\} \leqslant \frac{\left\|\Phi_{\mathrm{k}}^{(6)}(\psi(t))-\Phi_{\mathrm{k}}^{(6)}(\psi(0))\right\|_{\mu_{\beta}}^{2}}{\eta_{1}^{2}\left\|\left|\psi_{\mathrm{k}}\right|^{2}\right\|_{\mu_{\beta}}^{2}}$.

But $\Phi_{\mathrm{k}}^{(6)}(\psi(t))-\Phi_{\mathrm{k}}^{(6)}(\psi(0))=\int_{0}^{t} \dot{\Phi}_{\mathrm{k}}^{(6)}(\psi(s)) \mathrm{d} s$, so

$$
\left\|\Phi_{\mathrm{k}}^{(6)}(\psi(t))-\Phi_{\mathrm{k}}^{(6)}(\psi(0))\right\|_{\mu_{\beta}} \leqslant \int_{0}^{t}\left\|\dot{\Phi}_{\mathrm{k}}^{(6)}(\psi(s))\right\|_{\mu_{\beta}} \mathrm{d} s .
$$

Thanks to the invariance of the measure, the $L^{2}\left(\mu_{\beta}\right)$-norm is conserved under the dynamics, so for any $t \in \mathbb{R}$, we have

$$
\left\|\dot{\Phi}_{\mathrm{k}}^{(6)}(\psi(t))\right\|_{\mu_{\beta}}=\left\|\dot{\Phi}_{\mathrm{k}}^{(6)}(\psi(0))\right\|_{\mu_{\beta}}=\left\|\dot{\Phi}_{\mathrm{k}}^{(6)}\right\|_{\mu_{\beta}},
$$


and in particular we obtain

$$
\left\|\Phi_{\mathrm{k}}^{(6)}(\psi(t))-\Phi_{\mathrm{k}}^{(6)}(\psi(0))\right\|_{\mu_{\beta}} \leqslant t\left\|\dot{\Phi}_{\mathrm{k}}^{(6)}\right\|_{\mu_{\beta}} .
$$

So,

$$
\mu_{\beta}\left\{\psi:\left|\Phi_{\mathrm{k}}^{(6)}(\psi(t))-\Phi_{\mathrm{k}}^{(6)}(\psi(0))\right|>\eta_{1}\left\|\left|\psi_{\mathrm{k}}\right|^{2}\right\|_{\mu_{\beta}}\right\} \leqslant t^{2} \frac{\left\|\dot{\Phi}_{\mathrm{k}}^{(6)}\right\|_{\mu_{\beta}}^{2}}{\eta_{1}^{2}\left\|\left|\psi_{\mathrm{k}}\right|^{2}\right\|_{\mu_{\beta}}^{2}} \leqslant \eta_{2}
$$

for any $|t|<\frac{\eta_{1} \sqrt{\eta_{2}} \beta^{2+\frac{1}{10}}}{C}$, where we used lemmas 2.4 and 5.4. Using this result, we can study the variation of the $k$-action. In fact

$$
\begin{aligned}
& \mu_{\beta}\left\{\psi:\left.|| \psi_{\mathrm{k}}(t)\right|^{2}-\left|\psi_{\mathrm{k}}(0)\right|^{2}\left|>\eta_{1}\left\|\left|\psi_{\mathrm{k}}\right|^{2}\right\|_{\mu_{\beta}}\right\}\right. \\
& \leqslant \mu_{\beta}\left\{\psi:\left|\Phi_{\mathrm{k}}^{(6)}(\psi(t))-\Phi_{\mathrm{k}}^{(6)}(\psi(0))\right|>\frac{\eta_{1}}{3}\left\|\left|\psi_{\mathrm{k}}\right|^{2}\right\|_{\mu_{\beta}}\right\} \\
& +\mu_{\beta}\left\{\psi:\left.\left|\Phi_{\mathrm{k}}^{(6)}-\right| \psi_{\mathrm{k}}\right|^{2}\left|(t)>\frac{\eta_{1}}{3}\left\|\left|\psi_{\mathrm{k}}\right|^{2}\right\|_{\mu_{\beta}}\right\}\right. \\
& +\mu_{\beta}\left\{\psi:\left.\left|\Phi_{\mathrm{k}}^{(6)}-\right| \psi_{\mathrm{k}}\right|^{2}\left|(0)>\frac{\eta_{1}}{3}\left\|\left|\psi_{\mathrm{k}}\right|^{2}\right\|_{\mu_{\beta}}\right\}\right. \\
& \leqslant \frac{\eta_{2}}{2}+18 \frac{\left\|\Phi_{\mathrm{k}}^{(6)}-\left|\psi_{\mathrm{k}}\right|^{2}\right\|_{\mu_{\beta}}^{2}}{\eta_{1}^{2}\left\|\left|\psi_{\mathrm{k}}\right|^{2}\right\|_{\mu_{\beta}}^{2}} \leqslant \eta_{2}
\end{aligned}
$$

for any $\beta>\frac{C}{\eta_{1}^{\frac{10}{7}} \eta_{2}^{\frac{5}{7}}},|t|<\frac{\eta_{1} \sqrt{\eta_{2}} \beta^{2+\frac{1}{10}}}{C}$, where we used Chebyshev's inequality, the conservation of the Gibbs measure, (39) with $\delta=\frac{1}{\beta^{\frac{13}{10}}}$ and lemma 2.1 to estimate the second and the third term. Then theorem 1.1 is obtained by reformulating this inequality.

Proof of corollary 1.2. We consider two sequences $\eta_{1, k}:=\eta_{1}\left(1+k^{2}\right)^{\frac{1}{2}}$, $\eta_{2, k}:=\frac{\eta_{2}}{\left(1+k^{2}\right)}\left(\sum_{j} \frac{1}{1+j^{2}}\right)^{-1}$.

For any $k \in \mathbb{Z}$ and any $\alpha<1 / 2$, we define

$$
\mathcal{J}_{\alpha, k}:=\left\{\psi:\left.|| \psi_{k}(t)\right|^{2}-\left|\psi_{k}(0)\right|^{2} \mid \leqslant \frac{\eta_{1}}{\left(1+k^{2}\right)^{\alpha} \beta}\right\} .
$$

Using theorem 1.1, one has

$$
\begin{aligned}
\mu_{\beta}\left(\mathcal{J}_{\alpha, k}^{c}\right) & \leqslant \mu_{\beta}\left\{\psi:\left.|| \psi_{k}(t)\right|^{2}-\left|\psi_{k}(0)\right|^{2} \mid>\frac{\eta_{1}}{\left(1+k^{2}\right)^{\frac{1}{2}} \beta}\right\} \\
& =\mu_{\beta}\left\{\psi:\left.|| \psi_{k}(t)\right|^{2}-\left|\psi_{k}(0)\right|^{2} \mid>\frac{\eta_{1, k}}{\left(1+k^{2}\right) \beta}\right\} \leqslant \eta_{2, k}
\end{aligned}
$$


for any $|t|<C^{\prime} \eta_{1} \sqrt{\eta_{2}} \beta^{2+\varsigma}$.

Denote $\mathcal{J}_{\alpha}:=\cup_{k} \mathcal{J}_{\alpha, k}$, one has that

$$
\mu_{\beta}\left(\mathcal{J}_{\alpha}^{c}\right) \leqslant \sum_{k} \mu_{\beta}\left(\mathcal{J}_{\alpha, k}^{c}\right) \leqslant \eta_{2}
$$

\section{Acknowledgments}

We thank T Oh and N Burq for introducing us to the theory of Gibbs measure for PDEs. D Bambusi was partially supported by GNFM.

\section{Appendix A. Lemmas on Gaussian and Gibbs measure}

First, we recall that both Gibbs and Gaussian measures are constructed with a limit procedure starting from the "finite dimensional" measure which, in the Gaussian case, is defined by

$$
\begin{aligned}
& \mu_{\beta, g, N}:=\frac{\mathrm{e}^{-\frac{\beta}{2}\left\|\Pi_{N}(\psi)\right\|_{H^{1}}^{2}}}{Z_{g, N}(\beta)}=\frac{\mathrm{e}^{-\frac{\beta}{2} \sum_{|k|<N}\left(1+k^{2}\right)\left|\psi_{k}\right|^{2}}}{Z_{g, N}(\beta)}, \\
& Z_{g, N}(\beta):=\int_{\Pi_{N}\left(H^{s}\right)} \mathrm{e}^{-\frac{\beta}{2} \sum_{|k|<N}\left(1+k^{2}\right)\left|\psi_{k}\right|^{2}} \prod_{|k|<N} \mathrm{~d} \psi_{k} \mathrm{~d} \bar{\psi}_{k},
\end{aligned}
$$

where $\Pi_{N}\left(\left\{\psi_{k}\right\}_{k \in \mathbb{Z}}\right):=\left\{\psi_{k}\right\}_{|k|<N}$. (see [6]).

Lemma A.1. Let $N$ be an integer, $1>\gamma>0$, then there exists $\tilde{C}(\gamma)>0$ s.t. for any $\beta>0$ one has

$$
\frac{\int_{\Pi_{N}\left(H^{s}\right)} \prod_{|k|<N} \chi\left\{\left|\psi_{k}\right|<\frac{1}{\left(1+k^{2}\right)^{\frac{\gamma}{2} \sqrt{\beta}}}\right\}^{\mathrm{e}^{-\frac{\beta}{2}\left(1+k^{2}\right)\left|\psi_{k}\right|^{2}} \mathrm{~d} \psi_{k} \mathrm{~d} \bar{\psi}_{k}}}{Z_{g, N}(\beta)} \geqslant \mathrm{e}^{-\tilde{C}(\gamma)} .
$$

Moreover $\tilde{C}$ is independent of $N$.

Proof. Using the independence of all the variables, one gets

$$
\begin{aligned}
& \frac{\int_{\Pi_{N}\left(H^{s}\right)} \prod_{|k|<N} \chi\left\{\left|\psi_{k}\right|<\frac{1}{\left(1+k^{2}\right)^{\frac{\gamma}{2}} \sqrt{\beta}}\right\}^{-\frac{\beta}{2}\left(1+k^{2}\right)\left|\psi_{k}\right|^{2}} \mathrm{~d} \psi_{k} \mathrm{~d} \bar{\psi}_{k}}{Z_{g, N}(\beta)} \\
& =\prod_{|k|<N} \frac{2 \pi \int_{0}^{\infty} \chi_{\left\{\rho_{k}<\frac{1}{\left(1+k^{2}\right)^{\frac{\gamma}{2}} \sqrt{\beta}}\right\}^{-\frac{\beta}{2}\left(1+k^{2}\right) \rho_{k}^{2}} \rho_{k} \mathrm{~d} \rho_{k}}^{2 \pi \int_{0}^{\infty} \mathrm{e}^{-\frac{\beta}{2}\left(1+k^{2}\right) \rho_{k}^{2}} \rho_{k} \mathrm{~d} \rho_{k}}=\prod_{|k|<N} \frac{\int_{0}^{\frac{\left(1+k^{2}\right)^{1-\gamma}}{2}} \mathrm{e}^{-z_{k}} \mathrm{~d} z_{k}}{\int_{0}^{\infty} \mathrm{e}^{-z_{k}} \mathrm{~d} z_{k}}}{=\prod_{|k|<N}\left(1-\mathrm{e}^{-\frac{\left(1+k^{2}\right)^{1-\gamma}}{2}}\right) \geqslant \prod_{k \in \mathbb{Z}}\left(1-\mathrm{e}^{-\frac{\left(1+k^{2}\right)^{1-\gamma}}{2}}\right)}
\end{aligned}
$$




$$
=\mathrm{e}^{\sum_{|k| \in \mathbb{Z}} \log \left(1-\mathrm{e}^{-\frac{\left(1+k^{2}\right)^{1-\gamma}}{2}}\right)}=\mathrm{e}^{-\tilde{C}(\gamma)} .
$$

As $N \rightarrow \infty$, we get the following lemma

Lemma A.2. Let $\gamma$ be $1>\gamma>0$. Then, for any $\beta>0$, one has

$$
\begin{aligned}
& \left.\lim _{N \rightarrow \infty} \frac{\int_{\Pi_{N}\left(H^{s}\right)} \prod_{|k|<N} \chi\left\{\left|\psi_{k}\right|<\frac{1}{\left(1+k^{2}\right)^{\frac{\gamma}{2}} \sqrt{\beta}}\right\}^{\mathrm{e}^{-\frac{\beta}{2}\left(1+k^{2}\right)\left|\psi_{k}\right|^{2}} \mathrm{~d} \psi_{k} \mathrm{~d} \bar{\psi}_{k}}}{Z_{g, N}(\beta)}\right) \mathrm{d} \mu_{g, \beta} . \\
& =\int_{H^{s}}\left(\prod_{k \in \mathbb{Z}}^{\infty} \chi\left\{\left|\psi_{k}\right|<\frac{1}{\left(1+k^{2}\right)^{\frac{\gamma}{2}} \sqrt{\beta}}\right\}\right)
\end{aligned}
$$

Proof. For any $M>N, M \in \mathbb{N}$, one has

$$
\begin{aligned}
& \left.\int_{\Pi_{N}\left(H^{s}\right)} \prod_{|k|<N} \chi_{\left\{\left|\psi_{k}\right|<\frac{1}{\left(1+k^{2}\right)^{\frac{\gamma}{2}} \sqrt{\beta}}\right\}}\right\} \\
& \left.=\int_{\Pi_{M}\left(H^{s}\right)} \prod_{|k|<N} \chi_{\left\{\left|\psi_{k}\right|<\frac{1}{\left(1+k^{2}\right)^{\frac{\gamma}{2}} \sqrt{\beta}}\right\}}\right\}
\end{aligned}
$$

So, one has

$$
\begin{aligned}
& \lim _{M \rightarrow \infty} \int_{\Pi_{M}\left(H^{s}\right)} \prod_{|k|<N} \chi_{\left\{\left|\psi_{k}\right|<\frac{1}{\left(1+k^{2}\right)^{\frac{\gamma}{2}} \sqrt{\beta}}\right\}} \frac{\mathrm{e}^{-\frac{\beta}{2} \sum_{|k|<M}\left(1+k^{2}\right)\left|\psi_{k}\right|^{2}} \prod_{|k|<M} \mathrm{~d} \psi_{k} \mathrm{~d} \bar{\psi}_{k}}{Z_{g, M}(\beta)} \\
& \left.=\int_{H^{s}} \prod_{|k|<N} \chi_{\left\{\left|\psi_{k}\right|<\frac{1}{\left(1+k^{2}\right)^{\frac{\gamma}{2}} \sqrt{\beta}}\right.}\right\} \\
& \mathrm{d} \mu_{g, \beta} .
\end{aligned}
$$

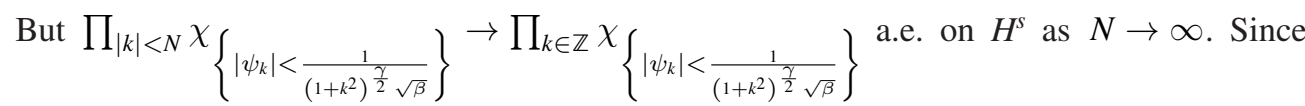
$1 \in L^{1}\left(H^{s}, \mu_{g, \beta}\right)$ and $\prod_{|k|<N} \chi\left\{\psi_{k} \mid<\frac{1}{\left(1+k^{2}\right)^{\frac{\gamma}{2}} \sqrt{\beta}}\right\}$
theorem,

$$
\begin{aligned}
& \lim _{N \rightarrow \infty} \int_{H^{s}} \prod_{|k|<N} \chi_{\left\{\left|\psi_{k}\right|<\frac{1}{\left(1+k^{2}\right)^{\frac{\gamma}{2} \sqrt{\beta}}}\right\}} \mathrm{d} \mu_{g, \beta}=\int_{H^{s}} \lim _{N \rightarrow \infty} \prod_{|k|<N} \chi_{\left\{\left|\psi_{k}\right|<\frac{1}{\left(1+k^{2}\right)^{\frac{\gamma}{2}} \sqrt{\beta}}\right\}^{2}} \mathrm{~d} \mu_{g, \beta} \\
& =\int_{H^{s}} \prod_{k \in \mathbb{Z}} \chi_{\left\{\left|\psi_{k}\right|<\frac{1}{\left(1+k^{2}\right)^{\frac{\gamma}{2} \sqrt{\beta}}}\right\}^{\mathrm{d}} \mu_{g, \beta} .}
\end{aligned}
$$


Remark A.1. From lemmas A.1 and A.2, we know that, if $1>\gamma>0$ and $\beta>0$, one has

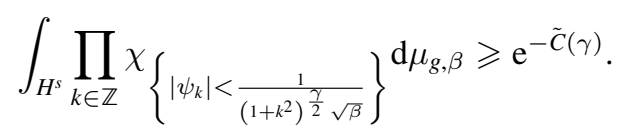

Lemma A.3. There exists a constant $\tilde{C}>0$ and $\beta^{*}>0$ s.t., for any $\beta>\beta^{*}$, one has

$$
1 \geqslant \int_{H^{s}} \mathrm{e}^{-\beta P} \mathrm{~d} \mu_{g, \beta} \geqslant \mathrm{e}^{-2 \tilde{C}}
$$

Proof. We remark that $P=\sum_{j=2}^{q} H_{2 j}=\sum_{j=2}^{q} \frac{c_{j}}{2 j}\|\psi\|_{L^{2 j}}^{2 j}$.

The first inequality is obvious.

We analyze now the second inequality. By the definition of $P$, if we fix $s_{1}$, by Sobolev's inequality $H^{s_{1}}(\mathbb{T}) \subset L^{r}(\mathbb{T})$ if $r \in\left[1, \frac{2}{1-2 s_{1}}\right]$. Therefore, choosing $\frac{q-1}{2 q}<s_{1}<\frac{1}{2}$, there exists a constant $C_{\text {sob }}$ s.t.

$$
\|\psi\|_{L^{2 j}}<C_{\mathrm{sob}}^{\frac{1}{2 j}}\|\psi\|_{H^{s_{1}}}, \quad j=2, \ldots, q .
$$

We fix $\frac{1}{2}+s_{1}<\gamma<1$, denote $D^{\prime}:=\sum_{j \in \mathbb{Z}} \frac{1}{\left(1+j^{2}\right)^{\gamma-s_{1}}}$, then we have:

$$
\begin{aligned}
& \int_{H^{s}} \mathrm{e}^{-\beta P} \mathrm{~d} \mu_{g, \beta} \geqslant \int_{H^{s}} \chi_{\left\{\|\psi\|_{H^{s_{1}}}^{2} \leqslant \frac{D^{\prime}}{\beta}\right\}} \mathrm{e}^{-\beta P} \mathrm{~d} \mu_{g, \beta}
\end{aligned}
$$

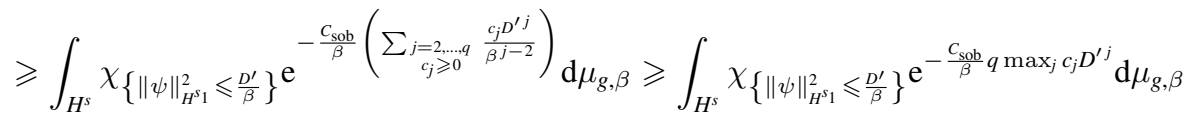

$$
\begin{aligned}
& \left.\geqslant \mathrm{e}^{-\frac{C_{\mathrm{sob}}}{\beta} q \max _{j} c_{j} D^{\prime j}} \int_{H^{s}} \prod_{k \in \mathbb{Z}} \chi_{\left\{\left|\psi_{k}\right|<\frac{1}{\left(1+k^{2}\right)^{\frac{\gamma}{2}} \sqrt{\beta}}\right.}\right\}^{\mathrm{d} \mu_{g, \beta}} \\
& \geqslant \mathrm{e}^{-\frac{C_{\mathrm{sob}}}{\beta} q \max c_{j} D^{\prime j}} \mathrm{e}^{-\tilde{C}(\gamma)} \geqslant \mathrm{e}^{-2 \tilde{C}(\gamma)},
\end{aligned}
$$

where the inequalities in the last line are true thanks to lemma A.2 and for $\beta$ sufficiently large.

Remark 16. $\mu_{\beta}$ is a good probability measure on $H^{s}$ since $\mu_{\beta}<\mu_{g, \beta}$ and $\mathrm{e}^{-2 \tilde{C}(\gamma)} \leqslant \frac{Z(\beta)}{Z_{g}(\beta)} \leqslant 1$

For the proof is sufficient to note that

$$
\int_{H^{s}} \mathrm{e}^{-\beta\left(\sum_{i=4}^{n} \frac{c_{i}}{i}\|\psi\|_{L^{i}}^{i}\right)} \mathrm{d} \mu_{g, \beta}=\frac{Z(\beta)}{Z_{g}(\beta)}
$$

Using this result, we can obtain lemma 2.1 to estimate the $L^{2}$-norm in the Gibbs measure with the norm in Gaussian measure.

Proof of lemma 2.1. We have 


$$
\|f\|_{\mu_{\beta}}^{2}=\int_{H^{s}}|f|^{2} \mathrm{~d} \mu_{\beta} \leqslant \frac{\int_{H^{s}}|f|^{2} \mathrm{~d} \mu_{g, \beta}}{\int_{H^{s}} \mathrm{e}^{-\beta P} \mathrm{~d} \mu_{g, \beta}}
$$

and, from lemma A.3,

$$
\|f\|_{\mu_{\beta}}^{2} \leqslant\|f\|_{g, \beta}^{2} \mathrm{e}^{2 \tilde{C}(\gamma)} .
$$

Proof of lemma 2.2. As above we fix $\frac{q-1}{2 q}<s_{1}<\frac{1}{2}$ and $\frac{1}{2}+s_{1}<\gamma<1$, we denote $D^{\prime}:=\sum_{j \in \mathbb{Z}} \frac{1}{\left(1+j^{2}\right)^{\gamma-s_{1}}}$, so we have:

$$
\begin{aligned}
& \|f\|_{\mu_{\beta}}^{2}=\int_{H^{s}}|f|^{2} \mathrm{~d} \mu_{\beta} \geqslant \int_{H^{s}}|f|^{2} \mathrm{e}^{-\beta P} \mathrm{~d} \mu_{g, \beta} \\
& \geqslant \int_{H^{s}}|f|^{2} \chi_{\left\{\|\psi\|_{H^{s_{1}}}^{2} \leqslant \frac{D^{\prime}}{\beta}\right\}} \mathrm{e}^{-\beta P} \mathrm{~d} \mu_{g, \beta} \\
& \geqslant \mathrm{e}^{-\frac{C_{\text {sob }}}{\beta} q \max _{j} c_{j} D^{\prime j}} \int_{H^{s}}|f|^{2} \chi_{\left\{\|\psi\|_{H^{s_{1}}}^{2} \leqslant \frac{D^{\prime}}{\beta}\right\}} \mathrm{d} \mu_{g, \beta} \\
& =\mathrm{e}^{-\frac{C_{\text {sob }}}{\beta} q \max _{j} c_{j} D^{\prime j}} \| f \chi_{\left\{\|\psi\|_{H^{s_{1}}}^{2} \leqslant \frac{D^{\prime}}{\beta}\right\} \|_{g, \beta} .}^{2} .
\end{aligned}
$$

We are now ready to give the proof of lemma 2.4, namely the estimate from below of the $L^{2}$-norm of the actions in Gibbs measure.

Proof of lemma 2.4. We fix $\frac{q-1}{2 q}<s_{1}<\frac{1}{2}$ and $\frac{1}{2}+s_{1}<\gamma<1$, we denote $D^{\prime}:=\sum_{j \in \mathbb{Z}} \frac{1}{\left(1+j^{2}\right)^{\gamma-s_{1}}}$, so

$$
\begin{array}{r}
\left\|\left|\psi_{\mathbf{k}}\right|^{2} \chi_{\left\{\|\psi\|_{H^{s}}^{2} \leqslant \frac{D}{\beta}\right\}}\right\|_{g, \beta}^{2} \geqslant \int_{H^{s}}\left|\psi_{\mathbf{k}}\right|^{4} \prod_{k \in \mathbb{Z}} \chi_{\left\{\left|\psi_{j}\right|<\frac{1}{\left(1+j^{2}\right)^{\frac{\gamma}{2} \sqrt{\beta}}}\right\}} \mathrm{d} \mu_{g, \beta} \\
=\lim _{N \rightarrow \infty} \frac{\left.\int_{\Pi_{N}\left(H^{s}\right)}\left|\psi_{\mathbf{k}}\right|^{4} \prod_{j \in \mathbb{Z}} \chi_{\left\{\left|\psi_{j}\right|<\frac{1}{\left(1+j^{2}\right)^{\frac{\gamma}{2}} \sqrt{\beta}}\right.}\right\}^{-\frac{\beta}{2} \sum_{|j|<N}\left(1+j^{2}\right)\left|\psi_{j}\right|^{2}} \prod_{|j|<N} \mathrm{~d} \psi_{j} \mathrm{~d} \bar{\psi}_{j}}{\int_{\Pi_{N}\left(H^{s}\right)} \mathrm{e}^{-\frac{\beta}{2} \sum_{j<N}\left(1+j^{2}\right)\left|\psi_{j}\right|^{2}} \prod_{|j|<N} \mathrm{~d} \psi_{j} \mathrm{~d} \bar{\psi}_{j}} .
\end{array}
$$

Using the independence of the variables, we have that (A.4) is equal to

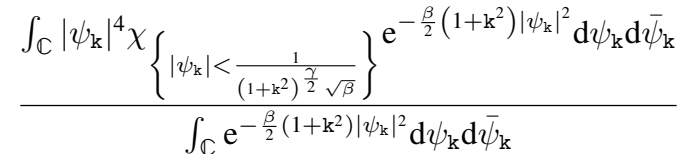

$$
\begin{aligned}
& \times \lim _{N \rightarrow \infty} \frac{\left.\int_{\prod_{N-1}^{k}\left(H^{s}\right)} \prod_{\substack{j \in \mathbb{Z} \\
j \neq \mathrm{k}}} \chi\left|\psi_{j}\right|<\frac{1}{\left(1+j^{2}\right)^{\frac{\gamma}{2} \sqrt{\beta}}}\right\}^{-\mathrm{e}^{-\frac{\beta}{2} \sum_{\substack{|j|<N \\
j \neq \mathrm{k}}}\left(1+j^{2}\right)\left|\psi_{j}\right|^{2}} \prod_{\substack{|j|<N \\
j \neq k}} \mathrm{~d} \psi_{j} \mathrm{~d} \bar{\psi}_{j}}}{\int_{\prod_{N-1}^{k}\left(H^{s}\right)} \mathrm{e}^{-\frac{\beta}{2} \sum_{\substack{|j|<N \\
j \neq k}}\left(1+j^{2}\right)\left|\psi_{j}\right|^{2}} \prod_{\substack{\begin{subarray}{c}{k j<N \\
j \neq \mathrm{k}} }} \\
{j}\end{subarray}} \mathrm{~d} \psi_{j} \mathrm{~d} \bar{\psi}_{j}},
\end{aligned}
$$


where $\Pi_{N}^{\mathrm{k}}$ is the Dirichlet projection onto the frequencies $\{|n|<N, n \neq \mathrm{k}\}$. Furthermore, since

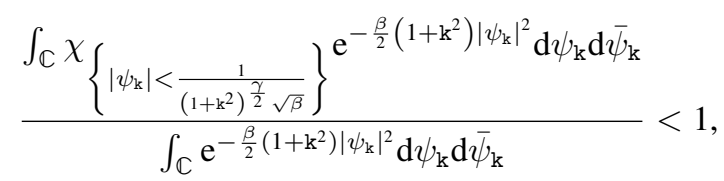

one has that (A.5) is lower than

$$
\begin{aligned}
& \frac{\left.\int_{\mathbb{C}}\left|\psi_{\mathrm{k}}\right|^{4} \chi_{\left\{\left|\psi_{\mathrm{k}}\right|<\frac{1}{\left(1+\mathrm{k}^{2}\right)^{\frac{\gamma}{2}} \sqrt{\beta}}\right.}\right\}^{\mathrm{e}^{-\frac{\beta}{2}\left(1+\mathrm{k}^{2}\right)\left|\psi_{\mathrm{k}}\right|^{2}} \mathrm{~d} \psi_{\mathrm{k}} \mathrm{d} \bar{\psi}_{\mathrm{k}}}}{\int_{\mathbb{C}} \mathrm{e}^{-\frac{\beta}{2}\left(1+\mathrm{k}^{2}\right)\left|\psi_{\mathrm{k}}\right|^{2}} \mathrm{~d} \psi_{\mathrm{k}} \mathrm{d} \bar{\psi}_{\mathrm{k}}} \\
& \times \lim _{N \rightarrow \infty} \frac{\left.\int_{\Pi_{N}\left(H^{s}\right)} \prod_{j \in \mathbb{Z}} \chi_{\left\{\left|\psi_{j}\right|<\frac{1}{\left(1+j^{2}\right)^{\frac{\gamma}{2}} \sqrt{\beta}}\right.}\right\}^{\mathrm{e}^{-\frac{\beta}{2} \sum_{|j|<N}\left(1+j^{2}\right)\left|\psi_{j}\right|^{2}} \prod_{|j|<N} \mathrm{~d} \psi_{j} \mathrm{~d} \bar{\psi}_{j}}}{\int_{\Pi_{N}\left(H^{s}\right)} \mathrm{e}^{-\frac{\beta}{2} \sum_{|j|<N}\left(1+j^{2}\right)\left|\psi_{j}\right|^{2}} \prod_{|j|<N} \mathrm{~d} \psi_{j} \mathrm{~d} \bar{\psi}_{j}} \\
& \left.\geqslant \frac{\int_{0}^{\frac{1}{\left(1+\mathrm{k}^{2}\right)^{\frac{\gamma}{2} \sqrt{\beta}}}} \rho_{\mathrm{k}}^{5} \mathrm{e}^{-\frac{\beta}{2}\left(1+\mathrm{k}^{2}\right) \rho_{\mathrm{k}}^{2}} \mathrm{~d} \rho_{\mathrm{k}}}{\int_{0}^{\infty} \rho_{\mathrm{k}} \mathrm{e}^{-\frac{\beta}{2}\left(1+\mathrm{k}^{2}\right) \rho_{\mathrm{k}}^{2} \mathrm{~d} \rho_{\mathrm{k}}}} \int_{H^{s}} \prod_{k \in \mathbb{Z}} \chi_{\left\{\left|\psi_{j}\right|<\frac{1}{\left(1+j^{2}\right)^{\frac{\gamma}{2} \sqrt{\beta}}}\right.}\right\}^{\mathrm{d} \mu_{g, \beta}} \\
& \geqslant \frac{4}{\beta^{2}\left(1+\mathrm{k}^{2}\right)^{2}} \int_{0}^{\frac{\left(1+\mathrm{k}^{2}\right)^{1-\gamma}}{2}} z_{\mathrm{k}}^{2} \mathrm{e}^{-z_{\mathrm{k}}} \mathrm{d} z_{k} \mathrm{e}^{-2 \tilde{C}(\gamma)} \\
& \geqslant \frac{\mathrm{e}^{-\tilde{C}(\gamma)}}{\beta^{2}\left(1+\mathrm{k}^{2}\right)^{2}} \int_{0}^{\frac{\left(1+\mathrm{k}^{2}\right)^{1-\gamma}}{2}} z_{\mathrm{k}}^{2} \mathrm{e}^{-z_{\mathrm{k}}} \mathrm{d} z_{\mathrm{k}} \geqslant \frac{\mathrm{e}^{-\tilde{C}(\gamma)}}{\beta^{2}\left(1+\mathrm{k}^{2}\right)^{2}} \int_{0}^{\frac{1}{2}} x^{2} \mathrm{e}^{-x} \mathrm{~d} x
\end{aligned}
$$

where in the last line we use lemma A.2. So, for $\beta$ large enough, using lemma 2.2, one has

$$
\begin{aligned}
& \left\|\left|\psi_{\mathrm{k}}\right|^{2}\right\|_{\mu_{\beta}}^{2} \geqslant \mathrm{e}^{-\frac{C_{\mathrm{sob}}}{\beta} q \max _{j} c_{j} D^{\prime q}}\left\|\left|\psi_{\mathrm{k}}\right|^{2} \chi_{\left\{\|\psi\|_{H^{s_{1}}}^{2} \leqslant \frac{D^{\prime}}{\beta}\right\}}\right\|_{g, \beta}^{2} \\
& \geqslant \mathrm{e}^{-\frac{C_{\text {sob }}}{\beta} q \max _{j} c_{j} D^{\prime q}} \frac{\mathrm{e}^{-\tilde{C}(\gamma)}}{\beta^{2}\left(1+\mathrm{k}^{2}\right)^{2}} \int_{0}^{\frac{1}{2}} x^{2} \mathrm{e}^{-x} \mathrm{~d} z_{\mathrm{k}}=\frac{C_{1}^{2}(\gamma)}{\beta^{2}\left(1+\mathrm{k}^{2}\right)^{2}} .
\end{aligned}
$$

The support of the Gaussian measure is described in the following lemma in which the main part is that we specify the dependence on $\beta$ of the rhs.

Lemma A.4. For any $s_{1}<\frac{1}{2}, a<\frac{1}{2}, M>0$ and $\beta$ large enough, there exists a constant $C>0$ s.t.

$$
\mu_{\beta}\left(\left\{\|\psi\|_{H^{s_{1}}}>M\right\}\right) \leqslant C \mathrm{e}^{-a \beta M^{2}}
$$


Proof. We consider

$$
\begin{aligned}
& \mathrm{e}^{a \beta M^{2}} \mu_{\beta}\left(\left\{\|\psi\|_{H^{s_{1}}}>M\right\}\right) \leqslant \mathrm{e}^{2 \tilde{C}} \mathrm{e}^{a \beta M^{2}} \mu_{g, \beta}\left(\left\{\|\psi\|_{H^{s_{1}}}>M\right\}\right) \\
& =\mathrm{e}^{2 \tilde{C}} \int_{\left\{\|\psi\|_{\left.H^{s_{1}}>M\right\} \cap H^{s}}\right.} \mathrm{e}^{a \beta M^{2}} \mathrm{~d} \mu_{g, \beta} \leqslant \mathrm{e}^{2 \tilde{C}} \int_{\left\{\|\psi\|_{\left.H^{s_{1}}>M\right\} \cap H^{s}}\right.} \mathrm{e}^{a\|\psi\|_{H^{s_{1}}}} \mathrm{~d} \mu_{g, \beta} \\
& \leqslant \mathrm{e}^{2 \tilde{C}} \int_{H^{s}} \mathrm{e}^{a \beta\|\psi\|_{H^{s_{1}}}} \mathrm{~d} \mu_{g, \beta}=\mathrm{e}^{2 \tilde{C}} \int_{H^{s}} \mathrm{e}^{a \beta \sum_{j}\left(1+j^{2}\right)^{s_{1}}\left|\psi_{j}\right|^{2}} \mathrm{~d} \mu_{g, \beta} \\
& =\mathrm{e}^{2 \tilde{C}} \frac{\int_{H^{s}} \mathrm{e}^{a \beta \sum_{j}\left(1+j^{2}\right)^{s_{1}}\left|\psi_{j}\right|^{2}-\frac{\beta}{2} \sum_{j}\left(1+j^{2}\right)\left|\psi_{j}\right|^{2}} \prod_{j} \mathrm{~d} \psi_{j} \mathrm{~d} \bar{\psi}_{j}}{\int_{H^{s}} \mathrm{e}^{-\frac{\beta}{2} \sum_{j}\left(1+j^{2}\right)\left|\psi_{j}\right|^{2}} \prod_{j} \mathrm{~d} \psi_{j} \mathrm{~d} \bar{\psi}_{j}} \\
& =\mathrm{e}^{2 \tilde{C}} \prod_{j} \frac{\int_{\mathbb{C}} \mathrm{e}^{a \beta\left(1+j^{2}\right)^{s_{1}}\left|\psi_{j}\right|^{2}-\frac{\beta}{2}\left(1+j^{2}\right)\left|\psi_{j}\right|^{2}} \mathrm{~d} \psi_{j} \mathrm{~d} \bar{\psi}_{j}}{\int_{\mathbb{C}} \mathrm{e}^{-\frac{\beta}{2}\left(1+j^{2}\right)\left|\psi_{j}\right|^{2}} \mathrm{~d} \psi_{j} \mathrm{~d} \bar{\psi}_{j}}
\end{aligned}
$$

Using the substitution $\psi_{j}=\frac{\sqrt{2 z_{j}}}{\sqrt{\beta\left(1+j^{2}\right)}} \mathrm{e}^{\mathrm{i} \theta_{j}}, z_{j} \in \mathbb{R}^{+}, \theta_{j} \in[0,2 \pi)$ and the fact that $\int_{\mathbb{R}^{+}} \mathrm{e}^{-z} \mathrm{~d} z=1$, one has that (A.7) is equal to

$$
\begin{aligned}
& \mathrm{e}^{2 \tilde{C}} \prod_{j} \int_{0}^{\infty} \mathrm{e}^{-\left(1-2 a\left(1+j^{2}\right)^{s_{1}-1}\right) z_{k}} \mathrm{~d} z_{k} \\
& =\mathrm{e}^{2 \tilde{C}} \prod_{j}\left(1+\frac{2 a}{\left(1+j^{2}\right)^{1-s_{1}}-2 a}\right)=C .
\end{aligned}
$$

Remark A.2. From the previous lemma, if $M$ goes to $+\infty$, we obtain that for any $s_{1}<\frac{1}{2}$,

$$
\mu_{\beta}\left(\left\{\|\psi\|_{H^{s_{1}}}=+\infty\right\}\right)=0 .
$$

In particular, we obtain that, for any $s_{1}>s, \mu_{\beta}\left(H^{s} \backslash H^{s_{1}}\right)=0$.

Proof of lemma 2.3. Having fixed $\beta$ large enough, $n>0$, and $a<\frac{\beta}{2}$, there exists a constant $C>0$ s.t. for any $x>C, x^{n}<\mathrm{e}^{a x^{2}}$, so, one has

$$
\begin{aligned}
& \int_{H^{s}}\|\psi\|_{H^{s_{1}}}^{n} \mathrm{~d} \mu_{g, \beta}<\int_{\left\{\|\psi\|_{H^{s_{1}}}<C\right\} \cap H^{s}}\|\psi\|_{H^{s_{1}}}^{n} \mathrm{~d} \mu_{g, \beta}+\int_{\left\{\|\psi\|_{\left.H^{s_{1}}>C\right\} \cap H^{s}}\right.} \mathrm{e}^{a\|\psi\|_{H^{s_{1}}}^{2} \mathrm{~d} \mu_{g, \beta}} \\
& \leqslant C^{n}+\int_{H^{s}} \mathrm{e}^{a\|\psi\|_{H^{s_{1}}}^{2}} \mathrm{~d} \mu_{g, \beta}=C^{n}+\prod_{j}\left(1+\frac{2 a}{\beta\left(1+j^{2}\right)^{1-s_{1}}-2 a}\right)<\infty,
\end{aligned}
$$

where in the last line we proceed as in lemma A.4. So we proved that $\|\psi\|_{H^{s_{1}}}^{n} \in L^{1}\left(H^{s}, \mathrm{~d} \mu_{g, \beta}\right)$. By lemma 2.1 we have that $\|\psi\|_{H^{s_{1}}}^{n} \in L^{1}\left(H^{s}, \mathrm{~d} \mu_{\beta}\right)$. 


\section{Appendix B. Technical lemmas}

\section{B.1. Proof of lemma 3.5}

We recall that, given a set $K$ of indices $\left(k_{1}, \ldots, k_{2 n}\right)$ with an even number of components, we denote

$$
K_{1}:=\left\{k_{1}, \ldots, k_{n}\right\}, \quad K_{2}:=\left\{k_{n+1}, \ldots, k_{2 n}\right\}
$$

Lemma B.1. Let $k \in \mathbb{Z}^{2 n}$ and $j \in \mathbb{Z}^{2 m}$ be 2 integer vectors, each one fulfilling the zero momentum condition and an $(M, \mathrm{k})$ admissible condition.

Assume that $K_{1} \cup J_{2}=K_{2} \cup J_{1}$, then there exist $x, y \in K_{1} \cup J_{2}$ and a constant $C$, s.t. $|x|,|y| \geqslant|\mathrm{k}| / C$. Furthermore $\{x, y\}$ is uniquely determined by $K_{1} \cup J_{2} \backslash\{x, y\}$.

Proof. For future reference we write the $(M, \mathrm{k})$ admissible conditions for the two vectors:

$$
\begin{aligned}
& \sum_{i=1}^{2 n} a_{i} k_{i}=\mathrm{k}, \\
& \sum_{i=1}^{2 n} b_{i} j_{i}=\mathrm{k} .
\end{aligned}
$$

We give now a recursive procedure in order to determine the elements $x, y$ in the statement.

From (B.1) there exists $l_{1}$ s.t. $\left|k_{l_{1}}\right| \geqslant|\mathrm{k}| / 2 n M$. By possibly interchanging $K_{1} \cup J_{2}$ with $K_{2} \cup J_{1}$ and reordering the indexes, we can always assume that $l_{1}=1$. So we have

$$
\left|k_{1}\right| \geqslant \frac{|\mathrm{k}|}{2 n M}, \quad a_{1} \neq 0 .
$$

In the following we will make several cases.

We look for the 'companion' of $k_{1}$ in $K_{2} \cup J_{1}$. We have two possibilities:

(A) It belongs to $J_{1}$ and therefore, by possibly reordering the indexes it is given by $j_{1}$ (thus we have $k_{1}=j_{1}$ ).

(B) It belongs to $K_{2}$ and therefore, by possibly reordering the indexes it is given by $k_{n+1}$ (thus we have $k_{1}=k_{n+1}$ ).

We begin by analyzing the case (A). We use the zero momentum condition on $k$ in order to compute $k_{1}$ as a function of the other components and we substitute in (B.1), which takes the form

$$
\sum_{i=2}^{n}\left(a_{i}-a_{1}\right) k_{i}+\sum_{i=1}^{n}\left(a_{i+n}+a_{1}\right) k_{i+n}=\mathrm{k} .
$$

Then there exists at least one of the $k_{i}$ 's which has modulus larger then a constant times $|\mathrm{k}|$. There are two possibilities

(A.1) It belongs to $K_{1}$, thus (up to reordering) it is given by $k_{n}$ : 


$$
\left|k_{n}\right| \geqslant \frac{|\mathrm{k}|}{2(n-1) M} \quad \& \quad a_{1} \neq a_{n} .
$$

(A.2) It belongs to $K_{2}$, thus (up to reordering) it is given by $k_{2 n}$ :

$$
\left|k_{2 n}\right| \geqslant \frac{|\mathrm{k}|}{2(n-1) M} \quad \& \quad a_{1} \neq-a_{2 n} .
$$

We analyze first (A.1). Consider the companion of $k_{n}$, there are two further possibilities:

(A.1.1) It belongs to $J_{1}$, call it $j_{m}$ (thus $k_{n}=j_{m}$ ),

(A.1.2) It belongs to $K_{2}$, call it $k_{2 n}$ (thus $k_{n}=k_{2 n}$ ).

We analyze (A.1.1). In this case, given $K_{1} \cup J_{2} \backslash\left\{k_{1}, k_{n}\right\}$ also $K_{2} \cup J_{1} \backslash\left\{j_{1}, j_{m}\right\}$ is fixed. Then (B.3) determines $k_{n}$ and then (B.1) determines $k_{1}$. This concludes the case (A.1.1).

We analyze now (A.1.2). Given $K_{1} \cup J_{2} \backslash\left\{k_{1}, k_{n}\right\}$ also $K_{2} \cup J_{1} \backslash\left\{j_{1}, k_{2 n}\right\}$ is fixed. So, also $J_{1} \cup J_{2} \backslash\left\{j_{1}\right\}$ is determined. Then, by the zero momentum condition on $j$ one determines $j_{1}=k_{1}$. Still one has to determine $k_{n}=k_{2 n}$. To this end one would like to use (B.3). This is possible if the coefficients of $k_{n}$ and $k_{2 n}$ do not cancel out. If this happens, then consider $k^{\prime}:=\left(k_{1}, \ldots, k_{n-1}, k_{n+1}, \ldots, k_{2 n-1}\right)$ and iterate the argument of situation (A) with it (which also fulfills the zero momentum condition). Iterating $n$ possibly decreases by one at each step. Since $k^{\prime}$ (and its iterates) has to fulfill an $(M, \mathrm{k})$ relation, which in particular is inhomogeneous, the procedure terminates with a nontrivial $k^{\prime}$ of dimension at least 2 . This concludes this case.

This concludes the analysis of (A.1).

We now analyze the case (A.2). We have two cases according to the position of the companion of $k_{2 n}$.

(A.2.1) It is $k_{n} \in K_{1}$ (thus $k_{n}=k_{2 n}$ )

(A.2.2) It is $j_{2 m} \in J_{2}$ (thus $j_{2 m}=k_{2 n}$ ).

The situation of the case (A.2.1) is identical to that of (A.1.2) and has already been analyzed.

We study now (A.2.2). Given $K_{1} \cup J_{2} \backslash\left\{k_{1}, j_{2 m}\right\}$ also $K_{1} \cup K_{2} \backslash\left\{k_{1}, k_{2 n}\right\}$ is determined. But, by the second of (B.5), (B.3) determines $k_{2 n}$. Then $k_{1}$ is determined by (B.1).

\section{This concludes the analysis of $(\mathrm{A})$.}

We come to (B). Substituting $k_{1}=k_{n+1}$ in (B.1) we get

$$
\left(a_{1}+a_{n+1}\right) k_{1}+\sum_{i=2}^{n}\left(a_{i} k_{i}+a_{i+n} k_{i+n}\right)=\mathrm{k} .
$$

We have two possibilities

$$
\begin{array}{ll}
\text { (B.1) } & -a_{1} \neq a_{n+1} \\
\text { (B.2) } & -a_{1}=a_{n+1}
\end{array}
$$

We analyze (B.1). We concentrate on $j$. By (B.2) there exists one of the $j_{i}$ 's which is 'big'. There are two cases 
(B.1.1) it belongs to $J_{1}$ and thus it is $\left|j_{1}\right| \geqslant|\mathrm{k}| / 2 m M$

(B.1.2) it belongs to $J_{2}$ and thus it is $\left|j_{2 m}\right| \geqslant|\mathrm{k}| / 2 m M$

Analyze (B.1.1). There are again two cases according to the companion of $j_{1}$

(B.1.1.1) It belongs to $K_{1}$, thus it is $k_{n}=j_{1}$.

(B.1.1.2) It belongs to $J_{2}$, thus it is $j_{m+1}=j_{1}$.

Analyze (B.1.1.1). Given $K_{1} \cup J_{2} \backslash\left\{k_{1}, k_{n}\right\}$ also $K_{2} \cup J_{1} \backslash\left\{k_{n+1}, j_{1}\right\}$ is determined. Thus also $J_{1} \cup J_{2} \backslash\left\{j_{1}\right\}$ is determined. So, from the zero momentum condition also $j_{1}=k_{n}$ is determined. From (B.6) also $k_{1}$ is determined.

We analyze (B.1.1.2). First we remark that given $K_{1} \cup J_{2} \backslash\left\{k_{1}, j_{2 n}\right\}$ also $K_{2} \cup J_{1} \backslash\left\{k_{n+1}, j_{n}\right\}$ is determined, thus $K_{1} \cup K_{2} \backslash\left\{k_{1}, k_{n+1}\right\}$ is determined, and then, by (B.6) also $k_{1}=k_{n+1}$ is determined. Then we have to determine one further large component.

Substituting $j_{1}=j_{m+1}$ in (B.2) one gets

$$
\sum_{i=2}^{m}\left(b_{i} j_{i}+b_{i+m} j_{i+m}\right)+\left(b_{1}+b_{m+1}\right) j_{1}=\mathrm{k} .
$$

We have two cases

(B.1.1.2.1) $b_{1}+b_{m+1} \neq 0$

(B.1.1.2.2) $b_{1}+b_{m+1}=0$

Case (B.1.1.2.1). Given $K_{1} \cup J_{2} \backslash\left\{k_{1}, j_{m+1}\right\}$ also $K_{2} \cup J_{1} \backslash\left\{k_{n+1}, j_{1}\right\}$ is determined. Thus also $J_{1} \cup J_{2} \backslash\left\{j_{1}, j_{m+1}\right\}$ is determined, but then one can use (B.7) to compute $j_{1}$. This concludes the analysis of this case.

Case (B.1.1.2.2). In this case (B.7) becomes a $(2 M, \mathrm{k})$ admissible condition for $j^{\prime}:=\left(j_{2}, \ldots, j_{m}, j_{m+2}, \ldots, j_{2 m}\right)$, which also fulfills the zero momentum condition. Thus one is again in the situation (B.1) but with $j^{\prime}$ in place of $j$. Iterating the construction one decreases $m$ at each step, and therefore the procedure terminates in a finite number of steps.

We come to the case (B.1.2). We distinguish two cases according to the position of the companion of $j_{2 m}$.

(B.1.2.1)It belongs to $K_{2}$, thus it is $k_{2 n}$.

(B.1.2.2)It belongs to $J_{1}$, thus it is $j_{2 m}$.

Case (B.1.2.1). Given $K_{1} \cup J_{2} \backslash\left\{k_{1}, j_{2 m}\right\}$ also $K_{2} \cup J_{1} \backslash\left\{k_{n+1}, k_{2 n}\right\}$ is determined. Thus also $J_{1} \cup J_{2} \backslash\left\{j_{2 m}\right\}$ is determined. Then by the zero momentum condition on $j$ also $j_{2 m}=k_{2 n}$ is determined and one can use (B.6) to determine $k_{1}$.

Case (B.1.2.2). By reasoning in a similar way, one determines $k_{1}=k_{n+1}$. Still one has to determine $j_{m}=j_{2 m}$ and this can be done exactly (up to a relabeling of the indexes) as in the case (B.1.1.2). It means that if $b_{1}+b_{m+1} \neq 0$ the argument is complete, otherwise we have to start a recursion as above in the case (B.1.1.2.2).

In the case (B.2), (B.6) becomes an $(M, \mathrm{k})$ admissible condition for $k^{\prime}:=$ $\left(k_{2}, \ldots, k_{n}, k_{n+2}, \ldots, k_{2 n}\right)$ which also fulfills the zero momentum condition. Thus the construction is repeated with $k^{\prime}$ in place of $k$ and after a finite number of steps the construction stops. 
We can now prove lemma 3.5.

Proof of lemma 3.5. The proof is similar to that of lemma 3.2. In the same way, we get an estimate analogous to (17), the only difference is that the sum is not on $\mathcal{T}$ but on the set of $(k, j)$ fulfilling the assumptions of lemma B.1. We denote this set by $\tilde{\mathcal{T}}$.

So, we estimate

$$
\sum_{(k, j) \in \tilde{\mathcal{T}}} \frac{1}{\prod_{i=1}^{n}\left(1+k_{i}^{2}\right)\left(1+j_{n+i}^{2}\right)} .
$$

If $\mathrm{k}=0$, then we can proceed exactly as in lemma 3.2.

If $\mathrm{k} \neq 0$, we note that at most $[(2 n !)]^{2}$ couples $(k, j)$ give the same set $K_{1} \cup J_{2}=K_{2} \cup J_{1}$. So using lemma B.1, we obtain

$$
\begin{aligned}
& \sum_{(k, j) \in \tilde{\mathcal{T}}} \frac{1}{\prod_{i=1}^{n}\left(1+k_{i}^{2}\right)\left(1+j_{n+i}^{2}\right)} \\
& \leqslant \frac{[(2 n) !]^{2}}{\left(1+\left(\frac{\mathrm{k}}{C}\right)^{2}\right)^{2}} \sum_{l_{1}, \ldots, l_{2 n-2}} \frac{1}{\prod_{t=1}^{2 m-2}\left(1+l_{t}^{2}\right)} \\
& \leqslant \frac{C}{\left(1+\mathrm{k}^{2}\right)^{2}}\left(\sum_{l} \frac{1}{\left(1+l^{2}\right)}\right)^{n-2} .
\end{aligned}
$$

\section{B.2. Estimate of the resonant part}

First, we introduce a lemma useful to estimate the measure of the resonant region.

Given $n \in \mathbb{N}$ and $k=\left(k_{1}, \ldots, k_{n}\right) \in \mathbb{Z}^{n}$, we denote by M the cardinality of $\operatorname{Supp}(k)$ and for any $\epsilon>0$, we define the non smooth cut-off function

$$
\chi(x)=\left\{\begin{array}{l}
0 \text { if }|x| \geqslant 1 \\
1 \text { if }|x|<1
\end{array}, \quad \chi_{\epsilon}(x):=\chi\left(\frac{x}{\epsilon}\right) .\right.
$$

Lemma B.2. Let $0<\epsilon, n \in \mathbb{N}, k=\left(k_{1}, \ldots, k_{n}\right) \in \mathbb{Z}^{n},\left\{a_{i}\right\}_{i=1}^{n} \in \mathbb{Z}^{n} \backslash\{0\}$. Then there exists a constant $C(n)>0$ s.t., denoting $\tilde{k}:=\min _{l \in \operatorname{Supp}(k), a_{l} \neq 0} k_{l}$ and $\tilde{a}$ the correspondent coefficient in $\left\{a_{i}\right\}_{i=1}^{n}$,

$$
\int_{\mathbb{R}_{+}^{M}}\left(\prod_{i=1}^{n} z_{k_{i}}\right) \chi\left(\sum_{i=1}^{n} a_{i} \frac{z k_{i}}{k_{i}^{2}}\right) \mathrm{e}^{-\sum_{l \in \operatorname{Supp}(k)} z_{l}} \prod_{l \in \operatorname{Supp}(k)} \mathrm{d} z_{l} \leqslant 4 \tilde{a} C(n) \tilde{k}^{2} \epsilon .
$$

Proof. We have that $z^{l} \mathrm{e}^{-z}<(2 l)^{l} \mathrm{e}^{-l} \mathrm{e}^{-\frac{z}{2}}<(2 n)^{n} \mathrm{e}^{-\frac{z}{2}}$, so, denoting by $I$ the left side of (B.12) and using the substitution $\frac{z_{l}}{2}=x_{l}$, we have

$$
I \leqslant C_{1}(n) \int_{\mathbb{R}_{+}^{M}} \chi\left(\sum_{i=1}^{n} 2 a_{i} \frac{x_{k_{i}}}{k_{i}^{2}}\right) \mathrm{e}^{-\sum_{l \in \operatorname{Sup}(k)} x_{l}} \prod_{l \in \operatorname{Supp}(k)} \mathrm{d} x_{l} .
$$


We denote $A(x):=\sum_{k_{i} \neq \tilde{k}} 2 a_{i} \frac{x_{k_{i}}}{k_{i}^{2}}$. So $I$ is bounded from above by

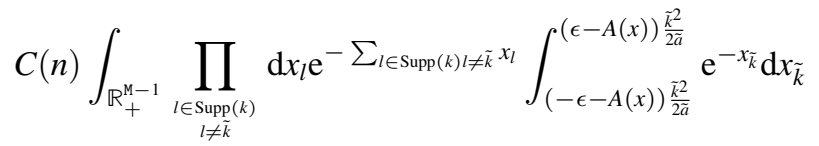

$$
\begin{aligned}
& <C(n) \int_{\mathbb{R}_{+}^{M-1}} \prod_{\substack{l \in \operatorname{Supp}(k) \\
l \neq \hat{k}}} \mathrm{~d} x_{l} \mathrm{e}^{-\sum_{l \in \operatorname{Supp}(k) l \neq \tilde{k}} x_{l}} \int_{(-\epsilon-A(x)) \frac{\hat{K}^{2}}{2 \tilde{a}}}^{(\epsilon-A(x)) \frac{\tilde{k}^{2}}{2 \bar{a}}} \mathrm{~d} x_{\tilde{k}}=4 \tilde{a} C(n) \tilde{k}^{2} \epsilon .
\end{aligned}
$$

Proof of lemma 5.2.

$$
\left\|\mathcal{R}_{6}^{R}\right\|_{g, \beta}^{2}=\left\|\sum_{k \in \mathcal{M}_{6}} Z_{6, k, \mathrm{k}}(\psi)\left(1-\rho\left(\frac{a_{k}(\psi)}{\delta}\right)\right)\right\|_{g, \beta}^{2},
$$

so

$\left\|\mathcal{R}_{6}^{R}\right\|_{g, \beta}^{2}$

$=\int_{H^{s}}\left(\sum_{k \in \mathcal{M}_{6}} Z_{6, k, \mathrm{k}}(\psi)\left(1-\rho\left(\frac{a_{k}(\psi)}{\delta}\right)\right)\right)\left(\sum_{j \in \mathcal{M}_{6}} \bar{Z}_{6, j, \mathrm{k}}(\psi)\left(1-\rho\left(\frac{a_{j}(\psi)}{\delta}\right)\right)\right) \mathrm{d} \mu_{\beta}$

$=\int_{H^{s}} \sum_{k, j \in \mathcal{M}_{6}} Z_{6, k, \mathrm{k}}(\psi) \bar{Z}_{6, j, \mathrm{k}}(\psi)\left(1-\rho\left(\frac{a_{j}(\psi)}{\delta}\right)\right)\left(1-\rho\left(\frac{a_{k}(\psi)}{\delta}\right)\right) \mathrm{d} \mu_{\beta}$.

As in lemma 3.5, for lemmas 3.1 and 2.3, we can use Lebesgue's dominated convergence to exchange the order between the integral and the series.

So (B.13) is equal to

$$
\sum_{k, j \in \mathcal{M}_{6}} \int_{H^{s}} Z_{6, k, \mathrm{k}}(\psi) \bar{Z}_{6, j, \mathrm{k}}(\psi)\left(1-\rho\left(\frac{a_{j}(\psi)}{\delta}\right)\right)\left(1-\rho\left(\frac{a_{k}(\psi)}{\delta}\right)\right) \mathrm{d} \mu_{\beta} .
$$

We analyze, now one single term of the series, namely:

$$
\begin{aligned}
& \tilde{Z}_{6, k}\left(\delta_{k_{1}, \mathrm{k}}+\delta_{k_{2}, \mathrm{k}}+\delta_{k_{3}, \mathrm{k}}-\delta_{k_{4}, \mathrm{k}}-\delta_{k_{5}, \mathrm{k}}-\delta_{k_{6}, \mathrm{k}}\right) \\
& \times \overline{\tilde{Z}}_{6, j}\left(\delta_{k_{1}, \mathrm{k}}+\delta_{k_{2}, \mathrm{k}}+\delta_{k_{3}, \mathrm{k}}-\delta_{k_{4}, \mathrm{k}}-\delta_{k_{5}, \mathrm{k}}-\delta_{k_{6}, \mathrm{k}}\right) \\
& \times \int \prod_{i=1}^{3} \psi_{j_{i}} \psi_{k_{3+i}} \bar{\psi}_{j_{3+i}} \bar{\psi}_{k_{i}}\left(1-\rho\left(\frac{a_{j}(\psi)}{\delta}\right)\right)\left(1-\rho\left(\frac{a_{k}(\psi)}{\delta}\right)\right) \mathrm{d} \mu_{\beta} .
\end{aligned}
$$

We remark that:

$$
a_{k}(\psi):=\left(\left|\psi_{k_{1}}\right|^{2}+\left|\psi_{k_{2}}\right|^{2}+\left|\psi_{k_{3}}\right|^{2}-\left|\psi_{k_{4}}\right|^{2}-\left|\psi_{k_{5}}\right|^{2}-\left|\psi_{k_{6}}\right|^{2}\right) .
$$


With the transformation $\psi=r \mathrm{e}^{\mathrm{i} \theta}$, denoted by $S_{k, j}:=\operatorname{Supp}(k, j)$, the integral becomes

$$
\begin{aligned}
& \frac{\int_{r_{k} \in \mathbb{R}_{+}} \prod_{i=1}^{6} r_{j_{i}} r_{k_{i}}\left(1-\rho\left(\frac{\tilde{a}_{j}(r)}{\delta}\right)\right)\left(1-\rho\left(\frac{\tilde{a}_{k}(r)}{\delta}\right)\right) \mathrm{e}^{-\beta \sum_{l \in S_{k, j}}\left(1+l^{2}\right) r_{l}^{2}} \prod_{k \in S_{k, j}} r_{l} \mathrm{~d} r_{l}}{\prod_{l \in S_{k, j}} \int_{\mathbb{R}_{+}} \mathrm{e}^{-\beta\left(1+l^{2}\right) r_{l}^{2}} l_{k} \mathrm{~d} r_{l}} \\
& \times \frac{\int_{\theta_{k} \in[0,2 \pi]} \mathrm{e}^{\mathrm{i}\left(\theta_{j_{1}}+\theta_{j_{2}}+\theta_{j_{3}}+\theta_{k_{4}}+\theta_{k_{5}}+\theta_{k_{6}}-\theta_{j_{4}}-\theta_{j_{5}}-\theta_{j_{6}}-\theta_{k_{1}}-\theta_{k_{2}}-\theta_{k_{3}}\right)} \prod_{l \in S_{k, j}} \mathrm{~d} \theta_{l}}{\prod_{k l \in S_{k, j}} \int_{\theta_{l} \in[0,2 \pi]} \mathrm{d} \theta_{l}}
\end{aligned}
$$

where

$$
\tilde{a}_{k}(r):=\left(r_{k_{1}}^{2}+r_{k_{2}}^{2}+r_{k_{3}}^{2}-r_{k_{4}}^{2}-r_{k_{5}}^{2}-r_{k_{6}}^{2}\right)
$$

The only terms different from 0 are the terms where

$$
\theta_{j_{1}}+\theta_{j_{2}}+\theta_{j_{3}}+\theta_{k_{4}}+\theta_{k_{5}}+\theta_{k_{6}}=\theta_{j_{4}}+\theta_{j_{5}}+\theta_{j_{6}}+\theta_{k_{1}}+\theta_{k_{2}}+\theta_{k_{3}}
$$

or equivalently

$$
\left\{j_{1}, j_{2}, j_{3}, k_{4}, k_{5}, k_{6}\right\}=\left\{j_{4}, j_{5}, j_{6}, k_{1}, k_{2}, k_{3}\right\} .
$$

This implies that the integrals that survive have this form:

$$
\begin{aligned}
& \frac{\int_{r_{k} \in \mathbb{R}_{+}} r_{j_{1}}^{2} r_{j_{2}}^{2} r_{j_{3}}^{2} r_{k_{4}}^{2} r_{k_{5}}^{2} r_{k_{6}}^{2}\left(1-\rho\left(\frac{\tilde{a}_{j}(r)}{\delta}\right)\right)\left(1-\rho\left(\frac{\tilde{a}_{k}(r)}{\delta}\right)\right) \mathrm{e}^{-\beta \sum_{l \in S_{k, j}}\left(1+l^{2}\right) r_{l}^{2}} \prod_{l \in S_{k, j}} r_{l} \mathrm{~d} r_{l}}{\prod_{l \in S_{k, j}} \int_{\mathbb{R}_{+}} \mathrm{e}^{-\beta\left(1+l^{2}\right) r_{l}^{2}} r_{l} \mathrm{~d} r_{l}} \\
& =\frac{\int_{z_{k} \in \mathbb{R}_{+}} z_{j_{1}} z_{j_{2}} z_{j_{3}} z_{k_{4}} z_{k_{5}} z_{k_{6}}\left(1-\rho\left(\frac{\tilde{b}_{j}(z)}{\beta \delta}\right)\right)\left(1-\rho\left(\frac{\tilde{b}_{k}(z)}{\beta \delta}\right)\right) \mathrm{e}^{-\sum_{l \in S_{k, j}} z_{l}} \prod_{l \in S_{k, j}} \mathrm{~d} z_{l}}{\beta^{6}\left(1+j_{1}\right)^{2}\left(1+j_{2}\right)^{2}\left(1+j_{3}\right)^{2}\left(1+k_{4}\right)^{2}\left(1+k_{5}\right)^{2}\left(1+k_{6}\right)^{2} \prod_{l \in S_{k_{j}}} \int_{\mathbb{R}_{+}} \mathrm{e}^{-\sum_{l} z_{l}} \mathrm{~d} z_{l}}
\end{aligned}
$$

where

$$
\tilde{b}_{k}(z):=\left(\frac{z_{k_{1}}}{1+k_{1}^{2}}+\frac{z_{k_{2}}}{1+k_{2}^{2}}+\frac{z_{k_{3}}}{1+k_{3}^{2}}-\frac{z_{k_{4}}}{1+k_{4}^{2}}-\frac{z_{k_{5}}}{1+k_{5}^{2}}-\frac{z_{k_{6}}}{1+k_{6}^{2}}\right)
$$

We define the non smooth cut-off function $\chi(x)=\left\{\begin{array}{l}0 \text { if }|x| \geqslant \delta \beta \\ 1 \text { if }|x| \leqslant \delta \beta\end{array}\right.$

So we can estimate the integral with the following integral:

$$
\begin{aligned}
& \frac{1}{\beta^{6}\left(1+j_{1}\right)^{2}\left(1+j_{2}\right)^{2}\left(1+j_{3}\right)^{2}\left(1+k_{4}\right)^{2}\left(1+k_{5}\right)^{2}\left(1+k_{6}\right)^{2}} \\
& \times \int \prod_{i=1}^{3} z_{j_{i}} \prod_{l=4}^{6} z_{k_{l}} \chi\left(\tilde{b}_{j}(z)\right) \chi\left(\tilde{b}_{k}(z)\right) \mathrm{e}^{-\sum_{l \in S_{k, j}} z_{l}} \prod_{l \in S_{k, j}} \mathrm{~d} z_{l} .
\end{aligned}
$$

We would like to know more information on the arguments of the cutoff function that depend on the form of $Z_{6, k, \mathrm{k}}$ and $Z_{6, j, \mathrm{k}}$. 
Since in $\mathcal{R}_{6}^{R}$ there are only terms in which $\left\{k_{1}, k_{2}, k_{3}\right\} \neq\left\{k_{4}, k_{5}, k_{6}\right\}$, this implies also that there are only terms in which $k_{i} \neq k_{l}$ for $i=1,2,3, l=4,5,6$, since if there exists at least an in$\operatorname{dex} i \in\{1,2,3\}$, and index $l \in\{4,5,6\}$ s.t. $k_{i}=k_{l}$ this implies that $\left\{k_{1}, k_{2}, k_{3}\right\}=\left\{k_{4}, k_{5}, k_{6}\right\}$ and it is absurd.

In fact, without losing generality we can suppose that $k_{1}=k_{4}$, this means that $k_{2}+k_{3}=k_{5}+k_{6}$ and $k_{2}^{2}+k_{3}^{2}=k_{5}^{2}+k_{6}^{2}$, so $k_{2}=k_{5}$ and $k_{3}=k_{6}$ or $k_{2}=k_{6}$ and $k_{3}=k_{5}$, so $\left\{k_{1}, k_{2}, k_{3}\right\}=\left\{k_{4}, k_{5}, k_{6}\right\}$.

So one has $j_{i} \neq j_{l}$ and $k_{i} \neq k_{l} \quad j=1,2,3, \quad l=4,5,6$. Moreover we know that $\left\{j_{1}, j_{2}, j_{3}, k_{4}, k_{5}, k_{6}\right\}=\left\{j_{4}, j_{5}, j_{6}, k_{1}, k_{2}, k_{3}\right\} \quad$ this means $\left\{j_{1}, j_{2}, j_{3}\right\}=\left\{k_{1}, k_{2}, k_{3}\right\} \quad$ and $\left\{k_{4}, k_{5}, k_{6}\right\}=\left\{j_{4}, j_{5}, j_{6}\right\}$ and $\left\{j_{1}, j_{2}, j_{3}, j_{4}, j_{5}, j_{6}\right\}=\left\{k_{1}, k_{2}, k_{3}, k_{4}, k_{5}, k_{6}\right\}=\left\{j_{1}, j_{2}, j_{3}, k_{4}, k_{5}, k_{6}\right\}$

So, up to permutation of the indices, we have nine cases:

- if $j_{i} \neq j_{l}, k_{i} \neq k_{l}, \tilde{b}_{k}(z)=\tilde{b}_{j}(z)=\left(\frac{z_{j_{1}}}{1+j_{1}^{2}}+\frac{z_{j_{2}}}{1+j_{2}^{2}}+\frac{z_{j_{3}}}{1+j_{3}^{2}}-\frac{z_{k_{4}}}{1+k_{4}^{2}}-\frac{z_{k_{5}}}{1+k_{5}^{2}}-\frac{z_{k_{6}}}{1+k_{6}^{2}}\right)$,

- if $j_{i} \neq j_{l}, k_{4}=k_{5}, \tilde{b}_{k}(z)=\tilde{b}_{j}(z)=\left(\frac{z_{j_{1}}}{1+j_{1}^{2}}+\frac{z_{j_{2}}}{1+j_{2}^{2}}+\frac{z_{j_{3}}}{1+j_{3}^{2}}-2 \frac{z_{k_{4}}}{1+k_{4}^{2}}-\frac{z_{k_{6}}}{1+k_{6}^{2}}\right)$,

- if $j_{i} \neq j_{l}, k_{4}=k_{5}=k_{6}, \tilde{b}_{k}(z)=\tilde{b}_{j}(z)=\left(\frac{z_{j_{1}}}{1+j_{1}^{2}}+\frac{z_{j_{2}}}{1+j_{2}^{2}}+\frac{z_{j_{3}}}{1+j_{3}^{2}}-3 \frac{z_{k_{4}}}{1+k_{4}^{2}}\right)$,

- if $j_{1}=j_{2}, k_{i} \neq k_{l}, \tilde{b}_{k}(z)=\tilde{b}_{j}(z)=\left(\frac{2 z_{j_{1}}}{1+j_{1}^{2}}+\frac{z_{j_{3}}}{1+j_{3}^{2}}-\frac{z_{k_{4}}}{1+k_{4}^{2}}-\frac{z_{k_{5}}}{1+k_{5}^{2}}-\frac{z_{k_{6}}}{1+k_{6}^{2}}\right)$,

- if $j_{1}=j_{2}, k_{4}=k_{5}, \tilde{b}_{k}(z)=\tilde{b}_{j}(z)=\left(\frac{2 z_{j_{1}}}{1+j_{1}^{2}}+\frac{z_{j 3}}{1+j_{3}^{2}}-2 \frac{z_{k_{4}}}{1+k_{4}^{2}}-\frac{z_{k_{6}}}{1+k_{6}^{2}}\right)$,

- if $j_{1}=j_{2}, k_{4}=k_{5}=k_{6}, \tilde{b}_{k}(z)=\tilde{b}_{j}(z)=\left(\frac{2 z_{j_{1}}}{1+j_{1}^{2}}+\frac{z_{j_{3}}}{1+j_{3}^{2}}-3 \frac{z_{k_{4}}}{1+k_{4}^{2}}\right)$,

- if $j_{1}=j_{2}=j_{3}, k_{i} \neq k_{l}, \tilde{b}_{k}(z)=\tilde{b}_{j}(z)=\left(3 \frac{z_{j_{1}}}{1+j_{1}^{2}}-\frac{z_{k_{4}}}{1+k_{4}^{2}}-\frac{z_{k_{5}}}{1+k_{5}^{2}}-\frac{z_{k_{6}}}{1+k_{6}^{2}}\right)$,

- if $j_{1}=j_{2}=j_{3}, k_{4}=k_{5}, \tilde{b}_{k}(z)=\tilde{b}_{j}(z)=\left(3 \frac{z_{j_{1}}}{1+j_{1}^{2}}-2 \frac{z_{k_{4}}}{1+k_{4}^{2}}-\frac{z_{k_{6}}}{1+k_{6}^{2}}\right)$,

- if $j_{1}=j_{2}=j_{3}, k_{4}=k_{5}=k_{6}, \tilde{b}_{k}(z)=\tilde{b}_{j}(z)=\left(3 \frac{z_{j_{1}}}{1+j_{1}^{2}}-3 \frac{z_{k_{4}}}{1+k_{4}^{2}}\right)$.

We can resume all this cases writing

$$
\begin{aligned}
& \tilde{b}_{k}(z)=\tilde{b}_{j}(z)=\tilde{b}_{k j}(z) \\
& =\left(a_{1} \frac{z_{j_{1}}}{j_{1}^{2}}+a_{2} \frac{z_{j_{2}}}{1+j_{2}^{2}}+a_{3} \frac{z_{j_{3}}}{1+j_{3}^{2}}-a_{4} \frac{z_{k_{4}}}{1+k_{4}^{2}}-a_{5} \frac{z_{k_{5}}}{1+k_{5}^{2}}-a_{6} \frac{z_{k_{6}}}{1+k_{6}^{2}}\right)
\end{aligned}
$$

where $a_{i} \in\{0,1,2,3\}, \sum_{i=1}^{6} a_{i}=6$, and $\left\{a_{i}\right\}_{i=1}^{6}$ s.t. if there exists $i \in\{1,2,3\}$ s.t. $a_{i} \neq 1$, for any $l \in\{1,2,3\}, l \neq i$ s.t. $a_{l}=0, j_{i}=j_{l}$ and if there exists $i^{\prime} \in\{4,5,6\}$ s.t $a_{i^{\prime}} \neq 1$, for any $l^{\prime} \in\{4,5,6\}, l^{\prime} \neq i^{\prime}$ s.t. $a_{l^{\prime}}=0, k_{i^{\prime}}=k_{l^{\prime}}$. In this way we can write (B.17) as

$\frac{1}{\beta^{6} \prod_{i=1}^{3}\left(1+j_{i}^{2}\right)\left(1+k_{3+i}^{2}\right)} \int \prod_{i=1}^{3} z_{j_{i}} z_{k_{3+i}} \chi\left(\tilde{b}_{k j}(z)\right) \mathrm{e}^{-\sum_{l \in S_{k, j}} z_{l}} \prod_{l \in S_{k, j}} \mathrm{~d} z_{l}$

where $z_{i} \in \mathbb{R}_{+}$.

To obtain the norm of the resonant part, after studying the form of any terms of the series, we have to estimate the norm of every single term.

Let $N$ be an integer, then lemma B.2 shows that if there exists at least an index $i=1,2,3$, $a_{i} \neq 0$ s.t. $\left|j_{i}\right|<N$ or an index $l=4,5,6, a_{l} \neq 0$ s.t. $\left|k_{l}\right|<N$, then there exists $C_{1}>0$ s.t. (B.18) is bounded by 


$$
C_{1} \frac{\delta \beta N^{2}}{\prod_{i=1}^{3}\left(1+j_{i}^{2}\right)\left(1+k_{3+i}^{2}\right)} .
$$

If every $j_{i}$ and $k_{l}$ really present in the argument of the cut-off is bigger than $N$, we adopt another strategy, because the distance between the two hyper-planes becomes bigger and non comparable with $\delta \beta$, so the presence of the cut-off is not so essential, because the integral is not so different from the integral over all the space. However, if all the indices in the argument of the cut-off are bigger than $N$, the denominators $\beta^{6} \prod_{i=1}^{3}\left(1+j_{i}^{2}\right)\left(1+k_{3+i}^{2}\right)$ is small and this helps the convergence. Obviously, since there exists at least an index $j_{i}$ or $k_{i}$ equal to $\mathrm{k}$, this situation is possible only if $|\mathrm{k}| \geqslant N$.

We denote by $T_{\mathrm{k}}$ the set of $(k, j) \in \mathbb{Z}^{12}$ s.t. $\left\{j_{1}, j_{2}, j_{3}, k_{4}, k_{5}, k_{6}\right\}=\left\{k_{1}, k_{2}, k_{3}, j_{4}, j_{5}, j_{6}\right\}$, $\sum_{i=1}^{n} k_{i}=\sum_{i=n+1}^{2 n} k_{i}, \quad \sum_{i=1}^{n} j_{i}=\sum_{i=n+1}^{2 n} j_{i}$, and s.t. there exists at least an index $i \in\{1,2,3,4,5,6\}$ s.t. $k_{i}=\mathrm{k}$ and at least an index $l \in\{1,2,3,4,5,6\}$ s.t. $j_{l}=\mathrm{k}$.

So, if $\mathrm{k}<N$, we have

$$
\left\|\mathcal{R}_{6}^{R}\right\|_{g, \beta}^{2} \leqslant 9 C_{1} \frac{\delta \beta N^{2}}{\beta^{6}} \sum_{j, k \in T_{\mathrm{k}}} \frac{\left|\tilde{Z}_{6, j}\right|\left|\tilde{Z}_{6, k}\right|}{\prod_{i=1}^{3}\left(1+j_{i}^{2}\right)\left(1+k_{3+i}^{2}\right)} .
$$

Instead, if $\mathrm{k} \geqslant N$, we have that $\left\|\mathcal{R}_{6}^{R}\right\|_{g, \beta}^{2}$ is bounded by

$$
\begin{aligned}
& 9 C_{1} \frac{\delta \beta N^{2}}{\beta^{6}} \sum_{j, k \in T_{\mathrm{k}}} \frac{\left|\tilde{Z}_{6, j}\right|\left|\tilde{Z}_{6, k}\right|}{\prod_{i=1}^{3}\left(1+j_{i}^{2}\right)\left(1+k_{3+i}^{2}\right)} \\
& +\frac{9}{\beta^{6}} \sum_{\substack{j, k \in T_{\mathrm{k}, s} \\
\forall i\left|i_{i}\right|,\left|k_{i}\right| \geqslant N}} \frac{\left|\tilde{Z}_{6, j}\right|\left|\tilde{Z}_{6, k}\right|}{\prod_{i=1}^{3}\left(1+j_{i}^{2}\right)\left(1+k_{3+i}^{2}\right)} .
\end{aligned}
$$

We know also that for every $j$ in the sum there is an index $i$ s.t. $j_{i}=\mathrm{k}$ but, due to the null momentum condition, there must be at least an other index $l$ s.t. $\left|j_{l}\right| \geqslant \frac{|\mathrm{k}|}{5}$ and the same holds also for any $k$. Moreover, from lemma 3.3, $\left|\tilde{Z}_{6, j}\right|$ are uniformly limited by a constant. So, in both the cases, as in theorem 3.5 , we have

$$
\sum_{j, k \in T_{\mathrm{k}}} \frac{\left|\tilde{Z}_{6, j}\right|\left|\tilde{Z}_{6, k}\right|}{\prod_{i=1}^{3}\left(1+j_{i}^{2}\right)\left(1+k_{3+i}^{2}\right)} \leqslant \frac{C}{\left(1+k^{2}\right)^{2}} \sum_{l_{1}, l_{2}, l_{3}, l_{4}} \frac{1}{\prod_{i=1}^{4}\left(1+l_{i}^{2}\right)}
$$

and, choosing $0<\epsilon \ll 1$,

$$
\begin{gathered}
\sum_{j, k \in T_{\mathrm{k}} \mathrm{s.t \forall i}\left|j_{i}\right|,\left|k_{i}\right| \geqslant N} \frac{\left|\tilde{Z}_{6, j}\right|\left|\tilde{Z}_{6, k}\right|}{\prod_{i=1}^{3}\left(1+j_{i}^{2}\right)\left(1+k_{3+i}^{2}\right)} \leqslant \frac{C}{\left(1+k^{2}\right)^{2}} \sum_{l_{1}, l_{2}, l_{3}, l_{4} \forall i,\left|l_{i}\right|>N} \frac{1}{\prod_{i=1}^{4}\left(1+l_{i}^{2}\right)} \\
\leqslant \frac{C}{\left(1+k^{2}\right)^{2} N^{4-4 \epsilon}} \sum_{l_{1}, l_{2}, l_{3}, l_{4} \forall i,\left|l_{i}\right|>N} \frac{1}{\prod_{i=1}^{4}\left(1+l_{i}^{2}\right)^{\frac{1+\epsilon}{2}}} .
\end{gathered}
$$

One has $\sum_{l_{1}, l_{2}, l_{3}, l_{4} \forall i,\left|l_{i}\right|>N} \frac{1}{\prod_{i=1}^{4}\left(1+l_{i}^{2}\right)^{\frac{1+\tau}{2}}} \sim \frac{1}{N^{4 \epsilon}}$, so, we can take 


$$
\delta \beta N^{2}=\frac{1}{N^{4}}
$$

one has $N=\frac{1}{(\delta \beta)^{\frac{1}{6}}}$ and finally

$$
\delta \beta N^{2}=\frac{1}{N^{4}}=(\delta \beta)^{\frac{2}{3}}
$$

This implies that

$$
\left\|\mathcal{R}_{6}^{R}\right\|_{g, \beta}^{2} \leqslant \tilde{C} \frac{(\delta \beta)^{\frac{2}{3}}}{\beta^{6}\left(1+k^{2}\right)^{2}} .
$$

\section{ORCID iDs}

D Bambusi (1) https://orcid.org/0000-0003-3229-1570

A Maiocchi (1) https://orcid.org/0000-0001-9268-4611

\section{References}

[1] Bambusi D 1999 Nekhoroshev theorem for small amplitude solutions in nonlinear Schrödinger equations Math. Z. $230345-87$

[2] Bambusi D 2003 Birkhoff normal form for some nonlinear PDEs Commun. Math. Phys. 234 253-85

[3] Bambusi D, Carati A, Maiocchi A and Maspero A 2015 Some analytic results on the FPU paradox Hamiltonian Partial Differential Equations and Applications (Fields Institute Communications) vol 75 (Toronto, ON: Fields Institute for Research in Mathematical Sciences) pp 235-54

[4] Bambusi D, Delort J M, Grébert B and Szeftel J 2007 Almost global existence for Hamiltonian semilinear Klein-Gordon equations with small Cauchy data on Zoll manifolds Commun. Pure Appl. Math. 60 1665-90

[5] Bambusi D and Grébert B 2006 Birkhoff normal form for partial differential equations with tame modulus Duke Math. J. 135 507-67

[6] Bourgain J 1994 Periodic nonlinear Schrödinger equation and invariant measures Commun. Math. Phys. 166 1-26

[7] Bourgain J 2000 On diffusion in high-dimensional Hamiltonian systems and PDE J. Anal. Math. $801-35$

[8] Burq N, Gérard P and Tzvetkov N 2001 The Schrödinger equation on a compact manifold: Strichartz estimates and applications J. Équ. Dérivées Partielles 18 (Univ. Nantes, Nantes (Plestin-lesGrèves, 2001))

[9] Burq N, Gérard P and Tzvetkov N 2004 Strichartz inequalities and the nonlinear Schrödinger equation on compact manifolds Am. J. Math. 126 569-605

[10] Carati A 2007 An averaging theorem for Hamiltonian dynamical systems in the thermodynamic limit J. Stat. Phys. 128 1057-77

[11] Carati A and Maiocchi A 2012 Exponentially long stability times for a nonlinear lattice in the thermodynamic limit Commun. Math. Phys. 314 129-61

[12] Colliander J and Oh T 2012 Almost sure well-posedness of the cubic nonlinear Schrödinger equation below $L^{2}(\mathbb{T})$ Duke Math. J. $161367-414$

[13] De Roeck W and Huveneers F 2015 Asymptotic localization of energy in nondisordered oscillator chains Commun. Pure Appl. Math. 68 1532-68

[14] Giorgilli A and Galgani L 1978 Formal integrals for an autonomous Hamiltonian system near an equilibrium point Celestial Mech. 17 267-80 
[15] Huang G 2013 An averaging theorem for a perturbed KDV equation Nonlinearity 26 1599-621

[16] Huang G, Kuksin S and Maiocchi A 2015 Time-averaging for weakly nonlinear CGL equations with arbitrary potentials Hamiltonian Partial Differential Equations and Applications (Fields Institute Communications) vol 75 (Toronto, ON: Fields Institute for Research in Mathematical Sciences) pp 323-49

[17] Lebowitz J L, Rose H A and Speer E R 1988 Statistical mechanics of the nonlinear Schrödinger equation J. Statist. Phys. 50 657-87

[18] Maiocchi A, Bambusi D and Carati A 2014 An averaging theorem for FPU in the thermodynamic limit J. Stat. Phys. 155 300-22

[19] Poincaré H 1899 Les Méthodes Nouvelles de la Mécanique Céleste vol 3 (Paris: Gauthier-Villars)

[20] Thomann L and Tzvetkov N 2010 Gibbs measure for the periodic derivative nonlinear Schrödinger equation Nonlinearity 23 2771-91

[21] Tzvetkov N 2008 Invariant measures for the defocusing nonlinear Schrödinger equation Ann. Inst. Fourier 58 2543-604 